Journal of Statistical Physics, Vol. 22, No. 3, 1980

\title{
Phase Transitions and Reflection Positivity. II. Lattice Systems with Short-Range and Coulomb Interactions
}

\author{
Jürg Fröhlich, ${ }^{1}$ Robert B. Israel, ${ }^{2}$ Elliott H. Lieb, ${ }^{3}$ and Barry Simon ${ }^{3}$
}

Received June 29, 1979

\begin{abstract}
We discuss applications of the abstract scheme of part I of this work, in particular of infrared bounds and chessboard estimates, to proving the existence of phase transitions in lattice systems. Included are antiferromagnets in an external field, hard-core exclusion models, classical and quantum Coulomb lattice gases, and six-vertex models.
\end{abstract}

KEY WORDS : Phase transitions; reflection positivity; chessboard estimates; contours.

\section{INTRODUCTION}

This is the second paper in a series describing applications of reflection positivity (RP) to proving the existence of phase transitions in model systems. We exploit: (1) the Peierls chessboard argument first used by Glimm et al. ${ }^{(22)}$ and further developed by Fröhlich and Lieb ${ }^{(13)} ;(2)$ the method of infrared bounds, first used by Fröhlich et al. ${ }^{(17)}$ and extended to quantum systems by Dyson et $\mathrm{al}^{\left({ }^{(6)}\right.}$ Reviews of some of these ideas can be found in Refs. 8, 9, 18, 30,36 , and 49 . In paper $I^{(11)}$ of this series, ${ }^{4}$ we presented a general framework, and in a third paper, ${ }^{(12)}$ we give applications to quantum field theories. In this paper, we deal with short-range lattice models and Coulomb lattice gases. In I, we discussed long-range lattice models. A further application to a model of a liquid crystal has been found by Heilmann and Lieb ${ }^{(26)}$ and one to dipole lattice gases by Fröhlich and Spencer. ${ }^{(19)}$

Research partially supported by Canadian National Research Council Grant A4015 and U.S. National Science Foundation Grants PHY-77-18762, MCS-75-21684-A02, and MCS-78-01885.

${ }^{1}$ IHES, Bures-sur-Yvette, France.

${ }^{2}$ Department of Mathematics, University of British Columbia, Vancouver, Canada.

${ }^{3}$ Departments of Mathematics and Physics, Princeton University, Princeton, New Jersey.

${ }^{4}$ Erratum to paper $\mathrm{I}^{(11)}$ : The Mermin argument ${ }^{(40)}$ uses $J \geqslant 0$, so this hypothesis should be added to Theorem 5.2 of Ref. 11. On the other hand, the existence part of the theorem does not require $J \geqslant 0$. 
Typically, the proof of phase transitions by the method of infrared bounds involves three steps:

(IR1) Verify reflection positivity for a suitably defined reflection. In many of the models below the reflection is in planes through lattice sites rather than between lattice sites.

(IR2) Choose the quantity, typically the Fourier transform of a twopoint function, for which an infrared bound is to be proven, e.g., $\langle\hat{s}(p) \hat{s}(-p)\rangle$ $\leqslant\left(\beta E_{p}\right)^{-1}$, and evaluate $E_{p}$, the spin wave energy. Check that $\int E_{p}{ }^{-1} d p<\infty$.

(IR3) Complete the argument, typically by proving a sum rule on the Fourier transform. Often symmetry is useful in this last step, but it is not essential; see Ref. 17. For infrared bounds one needs some couplings crossing the plane of reflection.

Typically the proof of phase transitions by the Peierls chessboard method also involves three steps:

(PC1) Identical to (IR1).

(PC2) Choose some decomposition of the configuration space at a single site, or small number of sites, and let $P^{(1)}, \ldots, P^{(n)}$ be the corresponding projections. Let $P_{\alpha}^{(i)}$ be the $P^{(i)}$ associated to site $\alpha$. Use the Peierls chessboard estimate to show that for suitable $i$ and $j$, $\sup _{\alpha, \gamma}\left\langle P_{\gamma}^{(i)} P_{\alpha}^{(j)}\right\rangle \rightarrow 0$ as $\beta$, the inverse temperature, goes to infinity. Here $\langle\cdots\rangle$ is typically an infinite-volume limit of periodic be equilibrium states. To prove this, one typically must compare the energy per unit volume of a finite number of periodic configurations to the energy per unit volume of some ground state.

(PC3) Completion of the argument: Show that (PC2) implies long-range order. This is discussed below.

We will assume some familiarity with these methods either from paper I or some of the other references given. We expand on step (PC3) in a number of successively more complicated situations.

\subsection{Two States Related by a Symmetry}

This is the situation of the usual spin- $-\frac{1}{2}$ Ising model. By symmetry of the interaction and boundary conditions,

$$
\left\langle P_{\alpha}^{(1)}\right\rangle=\left\langle P_{\alpha}^{(2)}\right\rangle
$$

and since only two states are involved, $\left\langle P^{(1)}\right\rangle+\left\langle P^{(2)}\right\rangle=1$, so $\left\langle P_{\alpha}^{(1)}\right\rangle=$ $\left\langle P_{\alpha}^{(2)}\right\rangle=1 / 2$. Thus, once

$$
\sup _{\alpha, \gamma}\left\langle P_{\alpha}^{(i)} P_{\gamma}^{(j)}\right\rangle \equiv A^{(i, j)}
$$

is less than $1 / 4$ for $(i, j)=(1,2)$ we have that

$$
\lim _{\Lambda \rightarrow \infty}|\Lambda|^{-2} \sum_{\alpha, \gamma \in \Lambda}\left[\left\langle P_{\alpha}^{(i)} P_{\gamma}^{(j)}\right\rangle-\left\langle P_{\alpha}^{(i)}\right\rangle\left\langle P_{\gamma}^{(j)}\right\rangle\right] \neq 0
$$

implying that $\langle\cdots\rangle$ is not an ergodic state, i.e., multiple phases occur. 


\subsection{Two Important States Related by a Symmetry}

This is the situation of the anisotropic classical Heisenberg model ${ }^{(13)}$ or the $\phi_{2}^{4}$ field theory. ${ }^{(22)}$ There are now three regions of the single-site configuration space singled out (the two polar regions, call them 1 and 2, and the remainder of the sphere, call it 0 ). Equation (1.1) still holds and $A^{(i j)}$, given by (1.2), goes to zero for $(i j)=(01),(02),(12)$ as $\beta \rightarrow \infty$. Finally, by a chessboard estimate, $\left\langle P_{\alpha}^{(0)}\right\rangle \rightarrow 0$ so, by (1.1), $\left\langle P_{\alpha}^{(1)}\right\rangle \rightarrow 1 / 2$ and thus (1.3) still holds.

\subsection{Two Important States Not Related by a Symmetry}

A good example of this is the Pirogov-Sinai triangle model, which we discuss in Section 2. Here there are two important states $P^{(1)}$ and $P^{(2)}$ and a third state $P^{(0)}$ with

$$
\left\langle P_{\alpha}^{(0)}\right\rangle_{\beta, \mu} \rightarrow 0
$$

as $\beta \rightarrow \infty$, uniformly as $\mu$, an "external field parameter," varies through a suitable compact set $[a, b]$. Moreover, $A^{(i j)}$, given by (1.2), goes to zero uniformly in $\mu$ for $(i j)=(01),(02),(12)$. However, (1.1) no longer holds. We use a device going back to Ref. 8 (related to an idea in Ref. 17): Suppose that

$$
s(\mu) \equiv\left\langle P_{\alpha}^{(1)}\right\rangle_{B, \mu} \quad \text { and } \quad t(\mu) \equiv\left\langle P_{\alpha}^{(2)}\right\rangle_{\beta, \mu}
$$

obey $s(a) \rightarrow 0, t(b) \rightarrow 0$ as $\beta \rightarrow \infty$. Vary $\mu$ between $a$ and $b$ at fixed, large $\beta$. Either $s(\mu)$ varies continuously, in which case there is a range of $\mu_{0}$ with $s\left(\mu_{0}\right)>1 / 3, t\left(\mu_{0}\right)>1 / 3$ and hence at least two phases, by (1.3), or else $s(\mu)$ varies discontinuously, in which case, picking a $\mu_{0}$ where $s(\mu)$ is discontinuous, we get at least two phases, by taking (subsequence) limits $\langle\cdots\rangle_{\beta, \mu_{0}+0}$ and $\langle\cdots\rangle_{B, \mu_{0}-0}$. The main defect in this procedure is that we do not know which possibility occurs (although we note that if there is precisely one $\mu$ with multiple phases, the second is realized) nor do we obtain any kind of continuity of $\mu_{0}$ in $\beta$ (although typically one can compute $\lim _{\beta \rightarrow \infty} \mu_{0}$ ).

\subsection{Three Important States with Two Related by Symmetry}

A typical example is the Fisher stabilized antiferromagnet at critical external field: see Section 3. There are four regions $P^{(0)}, \ldots, P^{(3)},(1.4)$ and (1.1) hold, $s(a) \rightarrow 0$, and $w(\mu) \equiv\left\langle P_{\alpha}^{(3)}\right\rangle_{\beta, \mu} \rightarrow 0$ for $\mu=b$. We now proceed as in Section 1.3 to find either a point with $s\left(\mu_{0}\right)=t\left(\mu_{0}\right) \sim 1 / 3$ or else two states $\langle\cdots\rangle_{\mu_{0}-0}$ and $\langle\cdots\rangle_{\mu_{0}+0}$ with $\left\langle P^{(3)}\right\rangle_{\mu_{0}-0}$ small, $\left\langle P^{(1)}\right\rangle_{\mu_{0}-0}=\left\langle P^{(2)}\right\rangle_{\mu_{0}-0}$ and $\left\langle P^{(3)}\right\rangle_{\mu_{0}+0}$ large. By (1.3), $\langle\cdots\rangle_{\mu_{0}-0}$ is still not ergodic, so there are at least three states. 


\subsection{Many States Related by a Symmetry}

A typical example is the three-state Potts model discussed in Section 3. We cannot just use the lack of ergodicity, so instead we use:

Theorem 1.1. Let $\langle\cdots\rangle$ be a translation-invariant state which is a linear combination of $k$ or fewer ergodic states. Let $\left\{X_{\alpha}^{(i)}\right\}_{i=1}^{m}$ be a set of $m$ distinct observables and let

$$
M_{i j}=\lim _{\Lambda \rightarrow \infty}|\Lambda|^{-1} \sum_{\alpha, \gamma \in \Lambda}\left\langle X_{\alpha}^{(i)} X_{\beta}^{(j)}\right\rangle
$$

Then $M_{i j}$ is a matrix with rank at most $k$.

Remark. The limit in (1.5) always exists by the mean ergodic theorem.

Proof. Let $\langle\cdots\rangle=\sum_{r=1}^{k} \lambda_{r}\langle\cdots\rangle_{r}$ with $\langle\cdots\rangle_{r}$ ergodic. Then

$$
|\Lambda|^{-2} \sum_{\alpha, \gamma}\left\langle X_{\alpha}^{(i)} X_{\beta}^{(j)}\right\rangle_{r} \rightarrow\left\langle X_{\alpha}^{(i)}\right\rangle_{r}\left\langle X_{\alpha}^{(j)}\right\rangle_{r}
$$

so that

$$
M_{i j}=\sum_{r=1}^{k} \lambda_{r}\left\langle X_{\alpha}^{(i)}\right\rangle_{r}\left\langle X_{\alpha}^{(j)}\right\rangle_{r}
$$

is rank $k$ or fewer.

If now the $X^{(i)}$ are projections with $\sum P^{(i)}=1$, then $\sum_{j} M_{i j}=\left\langle P_{\alpha}^{(i)}\right\rangle$. If $A^{(i j)} \rightarrow 0($ as $\beta \rightarrow \infty)$ for all $i \neq j$, then $M_{i j}$ goes to zero off-diagonal as $\beta \rightarrow \infty$. If we can find an integer $l_{0}$ such that $l_{0}$ of the $\left\langle P_{\alpha}^{(i)}\right\rangle$ are all bigger than, say, $\left(2 l_{0}\right)^{-1}$, then $M$ will have an $l_{0} \times l_{0}$ block which is very small off-diagonal and $>\left(2 l_{0}\right)^{-1}$ on-diagonal; hence $M$ will have rank at least $l_{0}$, so there will be at least $l_{0}$ phases by Theorem 1.1. One way of realizing this possibility is to have a symmetry which implies that $l_{0} P$ 's all have the same expectation and a chessboard estimate which shows that the remaining $P$ 's are all small.

\subsection{Many Phases Without Symmetries}

As a final level of complication, we want to show how the scheme of Section 1.3 can be extended. We consider a situation with $k+1$ important states and $k$ external field parameters, say $\lambda_{1}, \ldots, \lambda_{k}$, varied in the region $0 \leqslant \lambda_{i} \leqslant 1, \sum_{i=1}^{k} \lambda_{i} \leqslant 1$. Let $\lambda_{k+1}=1-\sum_{1}^{k} \lambda_{i}$ and suppose that

$$
\lim _{\beta \rightarrow \infty}\left\langle P_{\alpha}^{(i)}\right\rangle_{\lambda_{j}}=0 \text { if } \lambda_{i}=0 \quad \text { (uniformly in the other } \lambda \text { 's) }
$$

We want to show that for $\beta$ large there is some $\lambda^{(0)}=\left(\lambda_{1}^{(0)}, \ldots, \lambda_{k+1}^{(0)}\right)$ and a state $\langle\cdots\rangle$ which is a convex linear combination of states which are limits of $\langle\cdots\rangle_{\beta, \lambda}$ as $\lambda \rightarrow \lambda^{(0)}$ so that $\left\langle P^{(i)}\right\rangle=\left\langle P^{(1)}\right\rangle, i=1, \ldots, k+1$. Thus, as in 
Section 1.5 , there will be at least $k+1$ phases. Without any change one can accommodate situations with some symmetries and fewer parameters needed. Fix $\beta$ large and let $\Delta_{k}$ be the simplex

$$
\left\{\left(\lambda_{1}, \ldots, \lambda_{k+1}\right): \quad \lambda_{i} \geqslant 0, \quad \sum_{1}^{k+1} \lambda_{i}=1\right\}
$$

Let $f: \Delta_{k} \rightarrow \Delta_{k}$ be given by

$$
\left[f\left(\lambda^{(0)}\right)\right]_{i}=\left\langle P^{(i)}\right\rangle_{\beta, \lambda_{0}} / \sum_{1}^{k+1}\left\langle P^{(j)}\right\rangle_{\beta, \lambda_{0}}
$$

Let $F\left(\lambda^{(0)}\right)$ be the convex set in $\Delta_{k}$ consisting of all limits of convex combinations of limits $\lim _{\lambda_{n} \rightarrow \lambda_{0}} f\left(\lambda_{n}\right)$. Then $F$ maps $\Delta_{k}$ into subsets of $\Delta_{k}$ so that $F\left(\lambda_{0}\right)$ is closed and convex and $F$ is semicontinuous in the sense that if $a_{n} \rightarrow a$ and $a_{n} \in F\left(\lambda_{n}\right)$ and $\lambda_{n} \rightarrow \lambda$, then $a \in F(\lambda)$. Moreover, by (1.6), $F$ is close to leaving each face of $\Delta_{k}$ fixed. We now used the following topological results essentially in Brézis. (2)

Theorem 1.2. Fix $k$. Let $\Delta_{k}$ be the $k$-simplex. Then there exists $\epsilon>0$ so that every map $F$ from $\Delta_{k}$ into $2^{\Delta_{k}}$ obeying:

(i) $F(x)$ is convex for all $x$;

(ii) if $a_{n} \rightarrow a, x_{n} \rightarrow x$, and $a_{n} \in F\left(x_{n}\right)$, then $a \in F(x)$;

(iii) for every generalized face $G$ of $\Delta_{k}, x \in G$ and $a \in F(x)$ implies $\operatorname{dist}(a, G)<\epsilon$;

has

$$
\left(\frac{1}{k+1}, \ldots, \frac{1}{k+1}\right) \in \bigcup_{x \in \Delta_{k}} F(x)
$$

If one notes that (ii) is equivalent to the statement that the graph $\{(x, a) \mid a \in F(x)\}$ is closed, then this theorem is equivalent to Theorem A3 proven in the Appendix. This proves the claim above about $k+1$ phases.

Finally we list the models treated in the remainder of the paper. In Section 2 we "warm up" with the elementary models: the triangle model of Pirogov and Sinai ${ }^{(42)}$ and a model of Ginibrestudied by Kim and Thompson. ${ }^{(32)}$ In Section 3 we consider various antiferromagnets: we recover Dobrushin's result ${ }^{(5)}$ on multiple equilibrium states in the Ising antiferromagnet (in the interior of the critical triangle) and extend this result to anisotropic, classical Heisenberg ferromagnets. When next-nearest-neighbor ferromagnetic coupling is added (Fisher stabilization) we obtain three equilibrium states at suitable external field (recovering a result of Pirogov and Sinai) and also multiple states in the classical, isotropic antiferromagnet in external field. Furthermore, we discuss a three-state Potts model recently studied by Schick and Griffiths. ${ }^{(46)}$ In Section 4 we treat lattice gases with nearest neighbor exclusion for the square, triangular, and hexagonal lattices, recovering results of Dobrushin, ${ }^{(5)}$ 
Heilmann, ${ }^{(25)}$ and Heilmann and Praestgaard. ${ }^{(27)}$ In Section 5 we consider classical and quantum Coulomb monopole gases. Typical is our result that in a lattice where each site can have charge $0,+1$, or -1 with Coulomb monopole forces, at low temperature and suitable fugacity there are three phases, one with mainly unoccupied sites and one with the $A$ (resp. $B$ ) sublattice occupied with positive charges and the $B$ (resp. $A$ ) sublattice occupied with negative charges. In Section 6 we discuss some six- and eight-vertex models. In Section 7 we describe a useful version of the chessboard Peierls argument which is applicable in particular to the Slawny ${ }^{(50)}$ model of a ferromagnet with an infinity of phases. Finally in Section 8 we describe a rather special model which in $v$ dimensions has nearest neighbor ferromagnetic coupling $J$ and next-nearest-neighbor antiferromagnetic coupling $J / 2(\nu-1)$. For this kind of model, discrete symmetries are only presumably broken in three or more dimensions and continuous symmetries only in five or more dimensions. At the end of the paper, we include an Appendix containing a topological result of some significance in applications to complicated models.

We note that our results for the triangular and hexagonal lattice suffer from the same defects noted in Ref. 11 for the face-centered cubic lattice: namely we cannot be certain that the infinite-volume state has reflection positivity unless there is a single state. Thus, typically we can only assert the existence of multiple phases and not of long-range order or number of phases; see Sections 3 and 4.

The methods presented in this series generally yield relatively accurate lower bounds on the transition temperature. If, in addition, some of the tricks of the trade, most especially that of Gallavotti and Miracle-Solé(21) are used, we would expect that, in the simplest models, our bounds are within about $20 \%$ of the exact critical temperature. In this paper, we will not push the arguments to the point of explicit lower bounds on transition temperatures, but we will be careful to get pretty good upper bounds on probabilities of contours which then could be used as an element of good bounds on transition temperatures.

\section{TWO SIMPLE MODELS : THE TRIANGLE ISING MODEL OF PIROGOV-SINAI AND GINIBRE'S MODEL}

As a warmup, we begin with two simple models. We are especially interested in illustrating the method for obtaining phase transitions without symmetry.

Model 2.1. This is a model of Ginibre, studied in mean field approximation by $\mathrm{Kim}$ and Thompson. ${ }^{(32)}$ The basic Hamiltonian $H$ in a finite volume $\Lambda$, a "simple cubic" torus, is

$$
H=\sum_{\langle\alpha \beta\rangle}\left(s_{\alpha}-s_{\beta}\right)^{2}+a \sum_{\alpha} s_{\alpha}^{2}+\mu \sum_{\alpha} s_{\alpha} ; \quad a>0
$$


where the first sum is over nearest neighbor pairs. Letting $h=-\frac{1}{2} \mu a^{-1}$, we can add a constant to $H$ and obtain

$$
H^{\prime}=H+\mathrm{const}=\sum_{\langle\alpha \beta\rangle}\left(s_{\alpha}-s_{\beta}\right)^{2}+a \sum\left(s_{\alpha}-h\right)^{2}
$$

The spins $s_{\alpha}$ are required to take integer values $0, \pm 1, \ldots$; we allow the possibility of a priori weights $w(n)$ for $\{s \mid s=n\}$, so that at inverse temperature $\beta$ the partition function is given by

$$
Z=\sum_{n_{\alpha} \in Z}\left[\prod_{\alpha \in \Lambda} w\left(s_{\alpha}\right)\right] \exp \left[-\beta H\left(s_{\alpha \alpha}\right)\right]
$$

convergent if $w(n) \leqslant \exp \left(A n^{B}\right)$, with $B<2$. Kim and Thompson studied three models: (a) $w(n)=1$, all $n$; (b) $w(n)=1,|n| \leq N: w(n)=0$ for $|n|>N$; and (c) $w(n)=1$ for $n \geqslant 0$ and $w(n)=0$ for $n<0$.

To find the phase structure at low temperatures, it is always useful to look at ground states, i.e., configurations minimizing $H$. From $\left(2.1^{\prime}\right)$ it is obvious that the ground state has $s_{\alpha,}=s^{(0)}$ for all $\alpha$, where $s^{(0)}$ is determined by minimizing $\left(s^{(0)}-h\right)^{2}$. Clearly, if $h \neq \pm \frac{1}{2}, \pm \frac{3}{2}, \ldots$, there is a unique such $s^{(0)}$, but at $h=k+\frac{1}{2}\left(k \in \mathbb{Z}\right.$ ) two values (namely $s^{(0)}=k, k+1$ ) minimize $H$. Thus at low temperatures, we expect that there are infinitely many $h_{k}(\beta)$ (near $k+\frac{1}{2}$ as $\beta \rightarrow \infty$ ) with multiple phases. We will prove this assuming $D \geqslant w(n) \geqslant \delta>0$ for all $n$ [without this assumption one can still show, as below, that if $w(n) \neq 0$ all $n$, then for $\beta>\beta_{k}$ there are multiple phases at some $h_{k}(\beta)$ with $\left|h_{k}-k-\frac{1}{2}\right|<\frac{1}{2}$, but $\beta_{k}$ may go to $\infty$ as $\left.k \rightarrow \pm \infty\right]$. In case $w(n) \neq 0$ for $|n| \leqslant N$ and $w(n)=0$ for $|n|>N$, we get transitions at $2 N$ points. Without loss we look at $k=0$.

We begin by noting that since $H$ has only nearest neighbor coupling, the model has RP about planes between lattice sites and orthogonal to the coordinate axes. Let $P_{\alpha}{ }^{ \pm}$be the projection onto $s_{\alpha} \gtrless \frac{1}{2}$. Let $\Lambda$ have sides $4 L \times$ $4 L \times \cdots 4 L$ and let $Q$ be the product $\prod_{\alpha \in \Lambda} P_{\alpha}^{q(\alpha)}$, where $q(\alpha)$ is independent of $\alpha_{2} \ldots, \alpha_{v}$ and takes the values $++--++-\ldots$ in the $\alpha_{1}$ direction. Clearly, by taking all spins to be either 0 or 1

$$
Z \geqslant \delta^{|\Lambda|} \exp [-\beta c(h)|\Lambda|]
$$

where $c(h)=\min \left(a h^{2}, a(h-1)^{2}\right)$. On the other hand,

$$
Z\langle Q\rangle \leqslant D^{|\Lambda|} \exp \left[-\frac{1}{2}|\Lambda| \beta-\beta d(h, \beta)|\Lambda|\right]
$$

where

$$
\exp [-\beta d(h, \beta)]=\sum_{n} \exp \left[-\beta a(h-n)^{2}\right]
$$


It is easy to see that uniformly for $\left|h-\frac{1}{2}\right|<\frac{1}{2}$ and $\beta>\beta_{0}, e^{-\beta a(h)} \leqslant$ $A(\beta) e^{-\beta c(h)}$ with $A\left(\beta_{0}\right) \rightarrow 1$ as $\beta_{0} \rightarrow \infty$. Thus, one obtains a uniform bound

$$
\langle Q\rangle \leqslant\left[\epsilon_{1}(\beta)\right]^{|\Lambda|}
$$

with $\epsilon_{1} \rightarrow 0$ as $\beta \rightarrow \infty$ uniformly in $\Lambda$ and $\left|h-\frac{1}{2}\right|<\frac{1}{2}$. (In passing, we note that one could be more explicit in the bounds by replacing $P^{ \pm}$by four $P$ 's, namely $P^{0}, P^{1}, P^{>}, P^{<}$corresponding to $s_{\alpha}=0,1, \geqslant 2, \leqslant-1$. There would then be six $Q^{\prime}$ s. The one for the pair $P^{0}, P^{I}$ could be estimated as above. The $Q$ with a $P^{<}$or $P^{>}$can be estimated by a chessboard estimate and an easy estimate on $\left\langle\prod_{\alpha \in \Lambda} P^{>}\right\rangle$or $\left\langle\prod_{\alpha \in \Lambda} P^{<}\right\rangle$.) Given (2.2), the chessboard Peierls argument (see Ref. 13 or Ref. 11) immediately implies that

$$
\left\langle P_{\alpha}{ }^{+} P_{\gamma}{ }^{-}\right\rangle \leqslant \epsilon_{2}(\beta)
$$

uniformly in $\alpha, \gamma, \Lambda$, and $h$ with $\left|h-\frac{1}{2}\right|<\frac{1}{2}$, where $\epsilon_{2}(\beta) \rightarrow 0$ as $\beta \rightarrow \infty$.

In case all $w_{n}=1$, we are now done, since by symmetry $\left\langle P_{\alpha}{ }^{+}\right\rangle=\left\langle P_{\alpha}{ }^{-}\right\rangle$ at $h=\frac{1}{2}$. Use the strategy of Section 1.1. In general, we need only note that for $h<\frac{1}{2}$

$$
\begin{aligned}
\left\langle P_{\alpha}^{+}\right\rangle & \leqslant\left\langle\prod_{\alpha \in \Lambda} P_{\alpha}^{+}\right\rangle^{1 /|\Lambda|} \quad \text { (a chessboard estimate) } \\
& \leqslant\left\{\sum_{n \geqslant 1} D \exp \left[-a \beta(n-h)^{2}\right]\right\} / \delta \exp \left(-a \beta h^{2}\right)
\end{aligned}
$$

goes to zero and for $h>\frac{1}{2},\left\langle P_{\sigma}{ }^{-}\right\rangle$goes to zero. Thus, by the strategy of Section 1.3, there is for $\beta \geqslant \beta_{0}$ a multiple phase point $h_{0}(\beta)$ with $h_{0}(\beta) \rightarrow \frac{1}{2}$ as $\beta \rightarrow \infty$. Notice that since the estimates on $\beta_{0}$ only depend on $a, \delta$, and $D$ we get a bound uniform in $k$ on $\beta_{k}$. Thus:

Theorem 2.1. Consider the Hamiltonian $H$ of (2.1) with integral spins and a priori weights $w_{n}$ obeying $0<\delta \leqslant w_{n} \leqslant D$. Then there is a $\beta_{0}$ depending only on $\delta, D$, and $a$ so that for $\beta>\beta_{0}$ there are infinitely many $h_{k}(\beta)$ where there are multiple phases. Moreover, $\left|h_{k}-k-\frac{1}{2}\right|<\frac{1}{2}$ and

uniformly in $k$.

$$
\lim _{\beta \rightarrow \infty} h_{k}(\beta)=k+\frac{1}{2}
$$

Since the above model is the first one considered, we have given full details. Henceforth, we will be briefer.

Model 2.2. (Pirogov-Sinai triangle model). Consider spins $\sigma_{\alpha}$ taking values \pm 1 (with equal a priori weights) on a two-dimensional "simple cubic" torus $\Lambda$. The Hamiltonian is

where

$$
-H=j \sum_{\langle\alpha \beta\rangle} \sigma_{\alpha} \sigma_{\beta}-k \sum_{\langle\alpha \beta \gamma\rangle} \sigma_{\alpha} \sigma_{\beta} \sigma_{\gamma}+h \sum_{\alpha} \sigma_{\alpha}
$$

$$
j, k, h \geqslant 0
$$


the first sum is over all nearest neighbor pairs, and the second sum is over all triples forming an isosceles right triangle with side 1 . This model has been considered by Pirogov and Sinai, ${ }^{(42)}$ whose results we recover below. The important fact about this model, both historically and conceptually, is that the usual up-down symmetry of the Ising model is absent. The usual Peierls argument is very difficult, but Pirogov and Sinai succeeded in treating the model by introducing the notion of "contour gas." RP makes this unnecessary.

The periodic state is not RP about the "usual" reflections midway between lattice planes orthogonal to the coordinate axes. It is, however, RP about reflections in these lattice planes themselves since $-H$ then has the form $\theta A+A$. Notice that this kind of reflection positivity is independent of the signs of $j, k, h$. However, the equality of the couplings of the four kinds of right triangles is critical (it is irrelevant for the Pirogov-Sinai argument); the equality of the horizontal and vertical $j$ 's is not important for RP. The type of chessboard estimate that holds with RP through the sites involves the dual lattice and is given by Theorem 4.3 of Ref. 11; see also the discussion of the AC model in Ref. 13. Given this estimate, we can say two things immediately about the possible ground states:

(i) Among the ground states must be one of the 16 states obtained by picking a configuration of a $2 \times 2$ block and extending it to be periodic with period 2.

(ii) Any other ground state must have the property that every $2 \times 2$ block is among those that yield a minimal energy when extended periodically as in (i).

Because of reflection and rotational symmetries, the 16 states fall into six types given by Table I. The quantity $e(n)$ is the energy per site, i.e., $\langle E\rangle /|\Lambda|$, for the state extended periodically.

From this table and (i) and (ii) above, one sees that as long as

$$
2 j+|4 k-h|>2 k+\frac{1}{2} h
$$

the only candidates for ground states are all plus or all minus and that at $h=4 k$ there is a change over from plus to minus. Thus, we imagine fixing $k, j$ with

$$
k<\frac{1}{2} j
$$

Table I. States for the PS Triangle Model

\begin{tabular}{lll|lll}
\hline$n$ & Block & $-e(n)$ & $n$ & Block & $-e(n)$ \\
\hline 1 & $+\ddagger$ & $2 j-4 k+h$ & 4 & $\pm \mp$ & $-2 j$ \\
2 & \pm \pm & $2 j+4 k-h$ & 5 & $\ddagger$ & $-2 k-\frac{1}{2} h$ \\
3 & $+\mp$ & 0 & 6 & $\pm \ddagger$ & $2 k+\frac{1}{2} h$ \\
\hline
\end{tabular}


and vary $h$ in a neighborhood of $h=4 k$. By (2.5), condition (2.4) holds for all such $h$. We now want to estimate the probability of a contour separating a set of plus spins from a set of minus spins. A contour of length $l$ runs through precisely $l$ points of the dual lattice. For each such point $i$ we let $F_{i}$ be the projection onto the state of the four neighboring spins forced by the contour. Using Theorem 4.3 of Ref. 11, we bound the probability of the contour by $l \gamma\left(F_{i}\right)$ 's and so the statistical weight of any contour of length $l$ is bounded by

$$
B^{l} \equiv\left[\max \left(\gamma\left(F_{i}\right)\right)\right]^{l}
$$

Let $-e_{\infty}=\max (-e(1),-e(2))$. Then, if $i$ is a vertex of a straight portion of contour, $\gamma\left(F_{i}\right)^{|\Lambda|}$ is the expectation of state 3 periodized; hence $\gamma\left(F_{i}\right) \leqslant$ $\exp \left[-\beta\left(e(3)-e_{\infty}\right)\right]$. Among the various corners, the worst one is

$$
\pm ?
$$

Replacing ? by + or - in each site and using chessboard again on each site, we see that the quantity $B$ of (2.6) is

$$
\begin{aligned}
B=\max & \left\{\exp \left[-\beta\left(e(3)-e_{\infty}\right)\right] ; \exp \left[-\beta\left(e(6)-e_{\infty}\right)\right]\right. \\
& \left.+\exp \left[-\beta\left(e(4)-e_{\infty}\right)\right]\right\}
\end{aligned}
$$

Under (2.5), $B \rightarrow 0$ uniformly as $\beta \rightarrow \infty$ for $h$ near $4 k$. Moreover, for $h>4 k$ (resp. $h<4 k$ ) $\left\langle P_{-}\right\rangle$(resp. $\left\langle P_{+}\right\rangle$) goes to zero as $\beta \rightarrow \infty$. By the strategy of Section 1.3, we obtain:

Theorem 2.2. Consider the model of (2.3) with $j, k$ fixed obeying (2.5). Then for all $\beta$ large, there is an $h(\beta)$ where multiple phases occur. Moreover, $h(\beta) \rightarrow 4 k$ as $\beta \rightarrow \infty$.

When (2.3) fails, one can still analyze the model by the above methods. If $-j>k>0$, there is a first-order phase transition between states 2 and 4 for $h \simeq 4(k+j)$, and if $j<k<0$, there is a transition between states 1 and 4 for $h \simeq 4(k-j)$.

One can ask what happens when (2.3) holds but (2.5) fails. We have nothing definite to report, but one can look at the ground state structure to get some idea of what is likely. The structure that results is very similar to that of the hard-core gas with both nearest and second nearest neighbor exclusion (see Section 4). If (2.5) fails, then (2.4) still holds for $|h|$ very large, but for $h \sim 4 k,(2.4)$ fails. In the region where (2.4) fails, there are four basic blocks: $A= \pm+, B=\mp+, C={ }_{+}^{+}$, and $D={ }_{+}^{+}$; and so by (i) above there are four ground states with period two in both directions. By (ii) above, ground states are precisely configurations obeying: 
(iii) In every $2 \times 2$ block of four spins, one is minus.

(iv) Each minus spin has all its nearest and next nearest neighbors plus.

There are numerous states: Any set of only $A$ and $B$ columns or only $C$ and $D$ columns or any set of only $A$ and $D$ rows or only $B$ and $C$ rows will be a ground state, and these are all the ground states. Since there are no ground states with $A$ and $C$ on the same row, one might think there could be some kind of long-range order; however, there are infinitely many configurations with $A$ and. $C$ on the same row, all with energy relative to the ground state uniformly bounded: for example, take a single column of $C$ 's imbedded in a "sea" of $D$ 's and change a finite chain of $D$ 's in a single row all to $A$ 's. The change in energy needed to insert the $A$ 's is bounded uniformly in the length. This suggests that in the region $h \sim 4 k$ there is no long-range order. Moreover, at the critical $h$ 's where the ground state shifts from type 6 blocks to type 1 blocks or to type 2 blocks, the configurations that arise in a chessboard Peierls argument are ground states. So, presumably, there are no first-order phase transitions as $h$ is varied when (2.5) fails, but there could well be a higher order phase transition. In that case, the phase diagram for $\beta$ large, $h \sim 4 k, j \sim 2 k$ would involve a shift from a line of first-order phase transitions to a line of higher order transitions.

\section{ANTIFERROMAGNETS}

We begin by considering nearest neighbor antiferromagnets. In zero external field, the classical models are equivalent to the corresponding ferromagnets, so the existence of multiple equilibrium states follows by the arguments of Peierls ${ }^{(41)}$ (for the Ising case) and Malyshev ${ }^{(39)}$ (for the classical Heisenberg case; see also Ref. 13). The quantum Heisenberg models are not equivalent, but the quantum antiferromagnet can be treated directly in zero external field; see Ref. 6 for the isotropic case, Ref. 13 for the two-dimensional anisotropic case. The first four models in this section involve antiferromagnets in external field. One no longer has RP under the reflection used in Ref. 6 (see Ex. 3.4 in Ref. 11), i.e., reflection in a plane between lattice sites combined with $\sigma_{\alpha} \rightarrow-\sigma_{\alpha}$, for the change of sign does not respect the external field. So one reflects in planes containing lattice sites. This introduces two unfortunate limitations. First, quantum spins are no longer allowed, since we have no results on RP for quantum systems when reflections are in planes containing sites. Second, one does not get useful infrared bounds without a next-nearestneighbor ferromagnetic coupling (see Model 3.4 below).

Model 3.1. (Nearest neighbor Ising antiferromagnet in external field) The basic Hamiltonian in $\nu$ dimensions is

$$
H=(1 / 2 v) \sum_{\langle\alpha \beta\rangle} \sigma_{\alpha} \sigma_{\beta}-h \sum_{\alpha} \sigma_{\alpha}
$$


the sum being over nearest neighbors. We will recover the result of Dobrushin ${ }^{(5)}$ that there are multiple phases in the triangle in the $\langle T, h\rangle$ plane with

$$
|h|+T / T_{0} \leqslant 1
$$

(we have made no attempt to compare our $T_{0}$ with his).

Merely for notational simplicity, we consider the case $\nu=2$. The periodic states are RP with respect to reflections in lines orthogonal to the coordinate axes and through the sites. Moreover, the formal infinite-volume Hamiltonian has the form $A+\theta A$ with respect to reflections in diagonal planes through the sites. The usual finite-volume cutoffs destroy this RP, but we can pick cutoffs which preserve this RP at the cost of losing the other RP; namely one takes a periodic box with sides at $45^{\circ}$. [One can produce a state which is RP in all these planes by the following special argument: first, by changing signs in the even sublattice, transform the model into a ferromagnet in staggered field. By a monotonicity argument ${ }^{(23)}$ and the GKS inequalities, the state in constant field is RP in all planes. By FKG inequalities (see Ref. 16), one can turn on the staggering and by monotonicity again get RP in all planes.]

As in Model 2.2, we must examine six periodic configurations for ground states. The values of $-e(n)$ can be obtained from Table II by setting $\epsilon=0$ ( $\epsilon$ is a parameter which is not relevant here but appears in Model 3.3 as a next-nearest-neighbor ferromagnetic coupling).

Clearly, for $|h|<1$ there are two ground states obtained by periodizing state 1 and also by translating the resulting state by one unit. Given $\alpha$, let $P_{\alpha}^{( \pm)}$denote the projection onto spin \pm 1 at $\alpha$. We claim that as long as

$$
\begin{gathered}
\beta(1-|h|) \geqslant T_{0}^{-1} \\
\left\langle P_{\alpha}( \pm) P_{\alpha+\gamma}\left(\mp(-1)^{|\gamma|}\right)\right\rangle<\frac{1}{4}
\end{gathered}
$$

To prove (3.3) we use a contour argument, but now we draw contours between nearest neighbor spins if they have the same sign. We now argue as in Model 2.2. Straight segments of contours contribute $\exp (-\beta / 2)$, while the worst corners contribute $\exp \left[-\frac{1}{2}(\beta-\beta|h|)\right]+\exp [-(\beta-\beta|h|)]$, so for $|h|>\frac{1}{2}$ the worst contours are ones with only corners (as one should expect!) and these are uniformly small in the region where $\beta(1-|h|)$ is uniformly large. Thus (3.2') implies (3.3).

Table II. States for the Antiferromagnet

\begin{tabular}{lll|ccl}
\hline$n$ & Block & $-e(n)$ & $n$ & Block & $-e(n)$ \\
\hline 1 & $\pm \mp$ & $\frac{1}{2}+2 \epsilon$ & 4 & $\ddagger \ddagger$ & $-2 \epsilon$ \\
2 & $\ddagger \ddagger$ & $-\frac{1}{2}+h+2 \epsilon$ & 5 & $\pm \ddagger$ & $\frac{1}{2} h$ \\
3 & $\pm=$ & $-\frac{1}{2}-h+2 \epsilon$ & 6 & \pm \pm & $-\frac{1}{2} h$ \\
\hline
\end{tabular}


Next, we note that if there is a unique equilibrium state, then it must of necessity be mixing, so that as $\gamma \rightarrow \infty,\left\langle P_{\alpha}(+) P_{\alpha+\gamma}(+)\right\rangle \rightarrow\left\langle P_{\alpha}(+)\right\rangle^{2}$ and $\left\langle P_{\alpha}(-) P_{\alpha+\gamma}(-)\right\rangle \rightarrow\left\langle P_{\alpha}(-)\right\rangle^{2}$. Since one of the squares is at least $1 / 4$ (since $\left\langle P_{\alpha}(+)\right\rangle$ is monotone in $h$, we know which is larger), (3.3) would fail. That is, (3.3) implies the existence of more than one equilibrium state. Noticing that $\left(3.2^{\prime}\right)$ is equivalent to (3.2), we have:

Theorem 3.1. The Ising model of (3.1) has multiple equilibrium states in the region (3.2) for suitable $T_{0}$.

Note that our proof has actually shown more, namely nondifferentiability of the pressure in staggered field.

Model 3.2. (Nearest neighbor, classical anisotropic Heisenberg antiferromagnet in external field.) One advantage of the chessboard Peierls argument is that it extends so easily to other single-spin distributions. We imagine replacing $\sigma_{\alpha}$ in Model 3.1 by $\sigma_{\alpha z}, \sigma_{\alpha} \sigma_{\beta}$ by $\sigma_{\alpha} \cdot \sigma_{\beta}$, and $\sigma$ is now a threevector constrained to lie on the ellipsoid $\sigma_{x}{ }^{2}+\sigma_{u}{ }^{2}+(1-\delta) \sigma_{z}{ }^{2}=1$ with $\delta>0$ and the natural induced measure. (This is equivalent to spherical $\sigma$ 's with anisotropic coupling). In the same way that Fröhlich and Lieb ${ }^{(13)}$ treat the zero-field model, we introduce a decomposition $P_{\alpha}(+), P_{\alpha}(-)$, and $P_{\alpha}(0)$ corresponding to the two polar caps and the remainder of the ellipsoid. By an elementary chessboard estimate

$$
\left\langle P_{\alpha}(0)\right\rangle<\frac{1}{3} \quad \text { for } \beta>\beta_{0}, \quad|h| \leqslant 1
$$

[Warning: The configuration needed to prove (3.4) is different from that in Ref. 13, for the RP used here is different (i.e., reflection through, not between sites); $\left\langle P_{\alpha}(0)\right\rangle_{\Lambda} \leqslant\langle Q\rangle^{1 / \Lambda \mid}$, where $Q$ is the projection onto $\prod_{\gamma} P_{\gamma}(0)$, the product being over only those $\gamma$ with each component even. In Ref. 13, all $\gamma$ arise. But $\langle Q\rangle^{1 /|\Lambda|}$ is small for this $Q$ also.]

Next we claim that for suitable $T_{0}$

$$
\left\langle P_{\alpha}( \pm) P_{\alpha+\delta}\left(\mp(-1)^{|\gamma|}\right)\right\rangle<\frac{1}{9}
$$

when (3.2') holds. This is proven as in Model 3.1, but now some extra configurations need to be considered in the contour estimation [put a contour around all $P_{\alpha}(0)$ 's and between two neighboring $P_{\alpha}(+)$ 's or $P_{\alpha}(-)$ 's]. Using (3.4), or more properly (3.4) with $1 / 3$ replaced by a much smaller number, each dual lattice site on a contour can be shown to contribute a small amount. Since (3.4) implies that $\left\langle P_{\alpha}(+)\right\rangle>\frac{1}{3}$ or $\left\langle P_{\alpha}(-)\right\rangle>\frac{1}{3}$, (3.5) proves the existence of multiple equilibrium states. Thus:

Theorem 3.2. The anisotropic, classical Heisenberg antiferromagnet in external field has multiple phases in the region (3.2), where $T_{0}$ depends on the amount of anisotropy. 
Model 3.3. (Fisher stabilized Ising antiferromagnet.) For simplicity we work in two dimensions. The ground state structure of the Hamiltonian $H$ of (3.1) as $h$ is varied is as follows. For $|h|<1$, there are two ground states and for $|h|>1$ clearly one. However, for $h= \pm 1$, there are three ground state blocks, and since one of them is given by 5 (or 6) in Table II we can build up infinitely many ground states as we did in Model 2.2 when (2.4) failed. Also, configurations of type 5 destroy any kind of chessboard Peierls argument. Fisher ${ }^{(7)}$ remarked that one can remove the infinite degeneracy by adding a next-nearest-neighbor ferromagnetic coupling, i.e., we take

$$
H=\frac{1}{4} \sum_{\langle\alpha \beta\rangle} \sigma_{\alpha} \sigma_{\beta}-h \sum \sigma_{\alpha}-\epsilon \sum_{\langle\alpha \beta\rangle} \sigma_{\alpha} \sigma_{\beta}
$$

where the last sum is over next nearest neighbors and $\epsilon>0$. Since $\epsilon>0$, the model still has both RP properties mentioned above (the RP we use in this model is the one in coordinate lines, which holds irrespective of the sign of $\epsilon$, but in Model 3.4 we use the diagonal RP). We will prove the following result, already obtained by Pirogov and Sinai ${ }^{(43,44)}$ :

Theorem 3.3. Fix $\epsilon>0$ in the Hamiltonian (3.6). Then for all $\beta>\beta_{0}$, there exist $h(\beta)$ so that for $h=h(\beta)$ there are at least three extremal equilibrium states. As $\beta \rightarrow \infty, h(\beta) \rightarrow 1$.

The basic block energy densities are given in Table II (see Ex. 3.1). Near $h=1$, only blocks 1 and 2 can occur and so there are only three ground states at $h=1$, in agreement with Fisher's remark. Cover $\Lambda$ with $\frac{1}{4} \Lambda, 2 \times 2$ squares and for each $2 \times 2$ square $a$ let $P_{\alpha}(i), i=1, \ldots, 16$, represent the projections onto the 16 possible configurations. Let $i=1,2,3$ correspond to ,$+- \mp \pm,++$, respectively. By an elementary chessboard estimate using Table II

$$
\lim _{\beta \rightarrow \infty}\left\langle P_{a}(i)\right\rangle=0 \quad \text { for } \quad i \geqslant 4
$$

uniformly in $\Lambda$ and $h \in\left[\frac{1}{2}, \frac{3}{2}\right]$. Moreover,

$$
\begin{aligned}
\lim _{\beta \rightarrow \infty}\left\langle P_{a}(3)\right\rangle=0 & \text { if } \quad h=\frac{1}{2} \\
\lim _{\beta \rightarrow \infty}\left\langle P_{a}(1)+P_{a}(2)\right\rangle=0 & \text { if } \quad h=\frac{3}{2}
\end{aligned}
$$

and of course by symmetry

$$
\left\langle P_{a}(1)\right\rangle=\left\langle P_{a}(2)\right\rangle
$$

If we can prove that

$$
\lim _{\beta \rightarrow \infty}\left\langle P_{a}(i) P_{b}(j)\right\rangle=0 \quad \text { for } \quad i \neq j, \quad i, j=1,2,3
$$

uniformly in $a, b, \Lambda, h$, then by the strategy of Section 1.4 the theorem will be proven. (In Section 1 we expressed results in terms of ergodic states and 
Cesaro averages; here we need to use extremal Gibbs states and mixing.) We prove (3.7) by drawing contours between $2 \times 2$ blocks with distinct $2 \times 2$ states. The contour surrounding $a$ or $b$ will have state $i(=1,2$, or 3$)$ on one side and some states (say $k$ ) on the other. We could attempt a chessboard argument with the full eight-spin configurations, $i$ next to $k$. Instead, we use a device that will be very useful below (the same device is used in Ref. 26): namely, we take a magnifying glass and pick out a convenient part of the eight-spin state.

Specifically, given a contour $\gamma$ of length $L$ with $i$ 's inside, we consider the $15^{L}$ possibilities obtained by specifying the possible states that touch the outside of the contour (actually, if $\gamma$ has obtuse corners, fewer than $15^{L}$ occur). Each of the $15^{L}$ possibilities so obtained will have the form

$$
\alpha \equiv\left\langle\prod_{I \in \gamma} P_{a_{I}}(i) P_{b_{I}}(j(I))\right\rangle
$$

where $I \in \gamma$ labels all dual lattice points in $\gamma$, and $a_{I}$ and $b_{I}$ are the $2 \times 2$ blocks inside and outside $\gamma$, respectively. Moreover $j(I) \neq i$. If $j(I) \geqslant 4$, we majorize $P_{a_{I}}(i) P_{b_{l}}(j(I))$ by $P_{b_{I}}(j(I))$. If $j(I)<4$, we majorize it by $P_{c}(k)$, where $c$ is the four middle spins of the eight-spin configuration. For example, if $i=++$ and $j(I)={ }_{+}^{+}+$with $a_{I}$ to the left of $b_{I}$, then $k={ }_{+}^{+}$. By checking out the possibilities one finds that the $k$ 's which arise are always 4 or more. Thus $\alpha$ is dominated by something of the form

$$
\left\langle\prod_{J \in Q} P_{J}(k(J))\right\rangle
$$

where $Q$ contains at least $L / 3$ distinct dual lattice sites (each $b$ can be a $b_{I}$ for three $I$ 's!) and $k(J) \geqslant 4$. Thus the probability of a given contour is dominated by $15^{L} a^{L}$ where $a^{3}=\exp \beta\left[\min _{n \geqslant 3}(-e(n))-\max _{n=1,2}(-e(n))\right] \rightarrow 0$ as $\beta \rightarrow \infty$ uniformly for $h \in\left[\frac{1}{2}, \frac{3}{2}\right]$. This proves (3.7) and thus Theorem 3.3.

Model 3.4. (Fisher stabilized isotropic classical Heisenberg antiferromagnet in external field.) The basic Hamiltonian is

$$
H=(1 / 2 v) \sum_{\langle\alpha \beta\rangle} s_{\alpha} \cdot s_{\beta}-\epsilon \sum_{\langle\alpha \beta\rangle} s_{\alpha \alpha} \cdot s_{\beta}-h \sum_{\alpha} s_{\alpha} \cdot \hat{z}
$$

where $s_{\alpha}$ is a unit three-vector, the first sum is over nearest neighbors, and the second is over next nearest neighbors. We will only consider $\epsilon \geq 0$. As already noted, one has formal reflection positivity about two kinds of hyperplanes. For this reason, to find the infinite-volume ground-state energy we need only consider configurations with two spin values $s$ and $s^{\prime}$, one at those lattice sites $\alpha$ with $(-1)^{\alpha}=1$ and the other at the remaining sites. Thus we want to maximize

$$
-e\left(s, s^{\prime}\right)=\frac{1}{2} h\left(s+s^{\prime}\right) \cdot \hat{z}-\frac{1}{2} s \cdot s^{\prime}
$$


A little vector calculus with Lagrange multipliers shows that the maximum values and configurations are given by [with $\left.s_{\perp}=\left(s_{x}, s_{y}\right)\right]$

for $0<h<2 \quad \max \left(-e\left(s, s^{\prime}\right)\right)=\frac{1}{2}+\frac{1}{4} h^{2}, \quad s^{\prime} \cdot \hat{z}=s \cdot \hat{z}=\frac{1}{2} h, \quad s_{\perp}=-s_{\perp}{ }^{\prime}$

for $h \geqslant 2 \quad \max \left(-e\left(s, s^{\prime}\right)\right)=-\frac{1}{2}+h, \quad s=s^{\prime}=\hat{z}$

We wish to note two things about (3.10)-(3.11): first, the critical value of $h$ is 2 , not 1 as it is in the Ising model. Second, in the case of the plane rotor model, this maximum occurs on a discrete set. One can thus prove by the arguments in Model 3.2 [using $P_{\alpha}( \pm)$ as the projection onto neighborhoods of $s_{\alpha z}=h / 2, s_{\alpha x}= \pm\left[1-(h / 2)^{2}\right]^{1 / 2}$ and $P_{\alpha}(0)$ the remaining region]:

Theorem 3.4. Consider the plane rotor model in two dimensions with Hamiltonian (3.8) and with $\epsilon \geqslant 0$ ( $\epsilon=0$ allowed!). For each $h, 0<h<2$, there exists $\beta_{c}(h, \epsilon)$ so that for $\beta>\beta_{c}(h, \epsilon)$ there are multiple equilibrium states.

Returning now to the classical Heisenberg model, the above energetic calculations and chessboard estimates can be used to show that the joint probability distribution of two nearest neighbor spins is more and more concentrated on the maximizing set. In particular for $h$ fixed strictly between 0 and 2 and any $\epsilon \geqslant 0$ :

$$
\begin{aligned}
\lim _{\beta \rightarrow \infty}\left\langle\left(s_{\alpha z}-h / 2\right)^{2}\right\rangle & =0 \\
\lim _{\beta \rightarrow \infty}\left\langle\left[\left|s_{\alpha \perp}\right|-\left(1-\frac{1}{4} h^{2}\right)^{1 / 2}\right]^{2}\right\rangle & =0 \\
\lim _{\beta \rightarrow \infty}\left\langle\left(s_{\alpha \perp}+s_{\gamma \perp}\right)^{2}\right\rangle & =0
\end{aligned}
$$

for nearest neighbors $\alpha, \gamma$; all limits for the torus state, uniform in $\Lambda$. It is now clear that the model looks very much like a plane rotor model, so in two dimensions we expect no long-range order, and suspect that one might be able to prove this by a Mermin ${ }^{(40)}$ argument. In three or more dimensions, we do expect long-range order even when $\epsilon=0$, but we can only prove it for $\epsilon \neq 0$ ! The difficulty involves limitations in proving RP and the fact that infrared bounds require couplings that cross the refecting planes. For this reason, we will require reflection in the diagonal planes, and we therefore pick boundary conditions which will respect RP in diagonals (e.g., periodic with boxes having sides at $45^{\circ}$ ). Since we lose reflection in site planes, we should remark that (3.12) can be proven using the chessboard estimates that come from diagonal RP. We also note that one can get from any $A$ sublattice site to any other $A$ sublattice site by repeated diagonal reflections. 
We consider

$$
\begin{aligned}
Z\left(h_{\alpha}\right)=\langle\exp [ & -\frac{\beta}{2 \nu} \sum_{\langle\alpha \beta\rangle} s_{\alpha} \cdot s_{\beta}+\beta h \sum_{\alpha} s_{\alpha \alpha} \cdot \hat{z} \\
& \left.\left.-\frac{\beta \epsilon}{2} \sum_{\langle\alpha \beta\rangle^{\prime}}\left(s_{\alpha}-s_{\beta}-h_{\alpha}-h_{\beta}\right)^{2}\right]\right\rangle
\end{aligned}
$$

Standard theory ${ }^{(17,11)}$ shows that $Z\left(h_{\alpha}\right) \leq Z(0)$ if we use reflection in diagonal planes. From there it is fairly easy, by following the standard theory, to obtain

$$
\left|\left\langle s_{\alpha} \cdot s_{0}\right\rangle-1\right| \leq \frac{2}{(2 \pi)^{v}}\left(\int \frac{d^{v} p}{E_{p}}\right) \frac{3}{2}(\beta \epsilon)^{-1}
$$

where $E_{p}=\nu-\sum \cos p_{i}$, the integral is over $\left|p_{i}\right| \leqslant \pi$, and where $\alpha$ is restricted to lie on the sublattice with $(-1)^{\alpha}=1$. Conditions (3.12) and (3.13) imply:

Theorem 3.5. Consider the classical Heisenberg Hamiltonian (3.8) with $\nu \geq 3, \epsilon>0$, and $0<h<2$. Then for $\beta>\beta_{c}(\epsilon, \nu, h)$ the periodic state is not mixing and the rotational symmetry about the $z$ axis is spontaneously broken.

We emphasize that our failure to handle the $\epsilon=0$ case shows once more the limitations of using RP to prove spontaneously broken symmetry!

Model 3.5. (The Schick-Griffiths model.) Schick and Griffiths ${ }^{(46)}$ have recently studied a model on a triangular lattice, predicting its phase diagram by a renormalization group calculation; we will prove part of their structure with RP methods. We emphasize that a complete analysis of the phase structure in the region where we can only analyze modulo a technical caveat is possible using the Pirogov-Sinai theory. ${ }^{(43,44)}$ Since this is the first model on a triangular lattice we have considered in this series, it is useful to begin with some generalities about such lattices, concentrating particularly on reflections and chessboard estimates. We begin with the infinite lattice. The triangular lattice is the set of vectors in $\mathbb{R}^{2}$ generated by two unit vectors making an angle of $60^{\circ}$. If bonds are drawn in linking nearest neighbor sites, the plane is divided into equilateral triangles. If one begins by labeling the vertices of one triangle $A, B, C$, then it is geometrically obvious that one can extend the labeling to the whole lattice in exactly one way so that each triangle has one vertex of each type. This divides the triangular lattice into three sublattices, called respectively the $A, B, C$ sublattices. If we use $i$ to label the "dual lattice," i.e., the set of triangles, then $A_{i}, B_{i}, C_{i}$ will denote the $A, B, C$ vertices of triangle $i$. Clearly the infinite triangular lattice is left invariant by reflections in the three sets of parallel lines obtained by extending lattice bonds. An important geometric fact about these reflections is that they leave each of the 
basic sublattices invariant. This has the important consequence that the hexagonal lattice that is obtained by dropping the $B$ sublattice typically has the same RP structure as the triangular lattice, and the analyses of models on the two lattices are closely related (see Remark 2 below).

There is some point in describing the above geometry algebraically, namely to understand the situation in finite volume. If $\mathbf{e}, \mathbf{f}$ are the two generating unit vectors, we use $\langle x, y\rangle$ to denote the vector $x \mathbf{e}+y \mathbf{f}$. The six nearest neighbors of $\langle 0,0\rangle$ are $\langle \pm 1,0\rangle,\langle 0, \pm 1\rangle$, and $\langle \pm 1, \mp 1\rangle$. For $\langle m, n\rangle \in \mathbb{Z}^{2}$, let $\alpha(\langle m, n\rangle)$ be the congruence class of $m+2 n \bmod 3$. Then $\alpha(X)=1$ or 2 for each neighbor of $\langle 0,0\rangle$, and thus if the $A, B, C$ sublattices are defined to be those with $\alpha(X)=0,1,2$, respectively, then neighboring lattice sites are in different sublattices. The invariance of the sublattices is easily checked algebraically. For example, if $r$ is reflection in the plane $y=0$, then $r \mathbf{e}=\mathbf{e}$ and $r \mathbf{f}=\mathbf{e}-f$, so $r(\langle x, y\rangle)=\langle x+y,-y\rangle$, and thus $\alpha(r\langle m, n\rangle)=m-$ $n \equiv m+2 n \bmod 3$.

Now consider imposing periodic boundary conditions on a rhomboidal region like that shown in Fig. 1; so, for example, the spins labeled 1 and 2 are regarded as neighbors in one row. One has the naive perception that the choice of two directions breaks the symmetry of the triangle model under rotations by $60^{\circ}$, and that no reflection symmetry will be retained since reflections in orthogonal coordinates are involved.

In fact, the naive perception is wrong. If one has an $L \times L$ rhombus, the $60^{\circ}$ rotational symmetry is always preserved, and one has reflection invariance in all three directions as long as $L$ is even; moreover, one has a breakup into $A, B, C$ sublattices as long as $L$ is divisible by three. To check these facts, we use the algebraic machinery introduced above. The rhombus should be regarded as equivalence classes in the lattice $\left(\simeq \mathbb{Z}^{2}\right)$ modulo some sublattice, namely the sublattice $S_{L}=\{\langle m L, n L\rangle: m, n \in \mathbb{Z}\}$. To see when the $A, B, C$

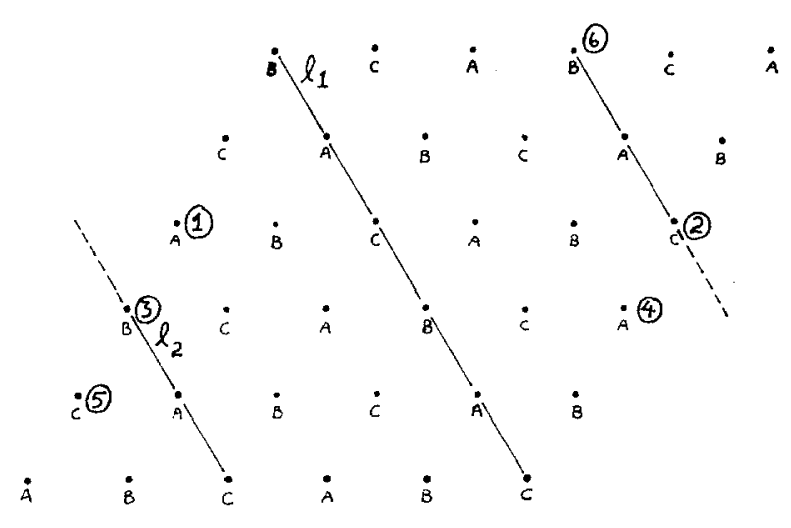

Fig. 1. Triangular lattice with periodic boundary conditions. 
substructure is preserved we ask when $\alpha(X+Y)=\alpha(X)$ for all $Y$ in $S_{L}$, i.e., when $(m+2 n) L$ is always congruent to zero mod 3 . This is true if and only if $L$ is divisible by three. In Fig. $1, L=6$ and the $A, B, C$ sublattices have been indicated. The rotation by $60^{\circ}$ takes $\mathbf{e}$ to $\mathbf{f}$ and $\mathbf{f}$ to $\mathbf{f}-\mathbf{e}$ and thus $\langle x, y\rangle \rightarrow$ $\langle-y, x+y\rangle$. This clearly leaves $S_{L}$ invariant and thus is definable on the quotient space. The same applies to the reflection $r$ above. This means that if $L$ is even, there will be reflection symmetry in three directions. For example, in Fig. 1 we indicate the two (!) lines $l_{1}$ and $l_{2}$ involved in a typical reflection in the nonobvious direction (we say two lines since 2 and 3 are neighboring spins and the two dotted segments are really the same segment). In attempting to obtain reflection positivity along these lines, $\mathfrak{A}_{+}$consists of the spins on the lines and in the regions with spins 4 and 5 (which are neighbors!), and $\mathfrak{U}_{-}$ is the remaining spins and the two lines. If $\theta$ is the reflection in line $l_{1}, \theta$ leaves the spins in $l_{1}$ fixed but not the spins in $l_{2}$; for example $\theta\left(\sigma_{3}\right)=\sigma_{6}$. This means that reflection positivity will fail-it even fails with no coupling between spins, for $\theta\left(\sigma_{3}-\sigma_{6}\right)=-\left(\sigma_{3}-\sigma_{6}\right)$ and thus $\left\langle\left[\theta\left(\sigma_{3}-\sigma_{6}\right)\right]\left[\sigma_{3}-\sigma_{6}\right]\right\rangle<0$.

Let $\langle\cdots\rangle_{\infty}$ be a limit of periodic states as $L \rightarrow \infty$. Since the infinitevolume state for uncoupled spins is RP, we would suppose that $\langle\cdots\rangle_{\infty}$ is RP if the interaction is of the form $A+\theta A$. We will thus make a working hypothesis that $\langle\cdots\rangle_{\infty}$ is $R P$ about all lattice lines. Below we will see what is implied if this working hypothesis is false.

To understand the chessboard estimates that hold in infinite volume, we begin by abstracting an argument of Seiler and Simon ${ }^{(47)}$ :

Lemma 3.6. Let $Y$ be a compact space and let $d \mu$ be a probability measure on $X=\chi_{n=-\infty}^{\infty} Y_{n}$ with each $Y_{n}$ a copy of $Y$. Suppose that $d \mu$ is invariant under the translation $y_{n} \rightarrow y_{n+2}$, i.e.

$$
\int f\left(y_{m}, y_{m+1}, \ldots, y_{m+k}\right) d \mu=\int f\left(y_{m+2}, y_{m+3}, \ldots, y_{m+k+2}\right) d \mu
$$

and reflection positive, i.e., for $f$ real-valued on $Y^{k}$,

$$
\begin{aligned}
& \int f\left(y_{1}, \ldots, y_{k}\right) f\left(y_{0}, y_{-1}, \ldots, y_{-k+1}\right) d \mu \geqslant 0 \\
& \int f\left(y_{0}, \ldots, y_{k-1}\right) f\left(y_{-1}, \ldots, y_{-k}\right) d \mu \geqslant 0
\end{aligned}
$$

For $g$ a function on $Y$, let

$$
\gamma(g)=\varlimsup\left[\int_{m=-L+1}^{L} g\left(y_{m}\right) d \mu\right]^{1 / 2 L}
$$

Then for $g_{m} \geqslant 0$

$$
\int \prod_{m=-M+1}^{M} g_{m}\left(y_{m}\right) d \mu \leqslant \prod_{m=M+1}^{M} \gamma\left(g_{m}\right)
$$


Proof. By using the Schwarz inequality about the point $m=\frac{1}{2}$, we can suppose that $g_{j}=g_{-j+1}$, and by homogeneity that $\left\|g_{j}\right\|_{\infty} \leqslant 1$. Suppose first that $M=1$. Then by reflecting successively in $m=\frac{3}{2}, \frac{7}{2}, \ldots, 2^{n}-\frac{1}{2}$ we see that (3.15) holds. Now suppose that (3.15) is known for $M=K-1$ and consider $M=K$. Then by repeated use of reflection in $m=-\frac{1}{2}$, we see that

$$
\int \prod_{m=-M+1}^{M} g_{m}\left(y_{m}\right) d \mu \leqslant a^{1-1 / 2^{n}} b_{n}
$$

where

$$
\begin{gathered}
a=\int g_{M}\left(y_{-M+1}\right) \cdots g_{2}\left(y_{-1}\right) g_{2}\left(y_{0}\right) \cdots g_{M}\left(y_{M-2}\right) d \mu \\
b_{n}=\left[\int h_{M} \prod_{n} g_{1}\left(y_{j}\right) d \mu\right]^{1 / 2^{n}}
\end{gathered}
$$

where $h_{M}$ is a product of $2 M-2$ of the $g$ 's and $\Pi_{n}$ is a product of $2^{n}$ successive sites. By induction, $a \leqslant \prod_{m \neq 0,1} \gamma\left(g_{m}\right)$, and clearly since $\left\|h_{M}\right\|_{\infty} \leqslant 1, b_{n} \leqslant$ $\left[\int \prod_{n}^{*} g_{1}\left(y_{j}\right) d \mu\right]^{1 / 2^{n}}$. Taking $n \rightarrow \infty$, (3.15) results.

Now use this lemma on $\langle\cdots\rangle_{\infty}$, supposing that it is RP. Let $F_{\alpha}$ be a function of the spins around a single triangle $\alpha$. We reflect successively in each of three directions using the lemma. What happens to a single triangle is shown in Fig. 2, where $r$ denotes one of the parallel lines about which one is about to reflect. In the end we get a very large number of factors, say $k$, of $l$ th roots of expectations of several parallel arrays of the type shown in Fig. 3. If we started with $L$ of the $F$ 's, then we have $l L$ triangles in the $k$ factors. What

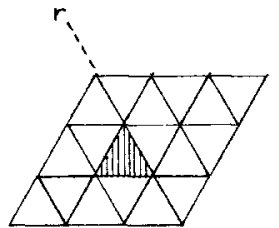

(a)

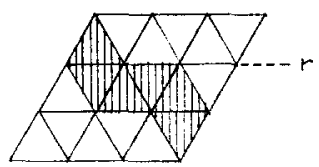

(c)

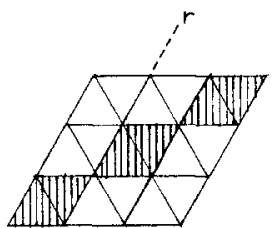

(b)

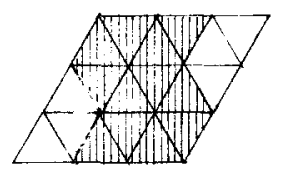

(d)

Fig. 2. Arrays produced by repeated reflection. 
Fig. 3. Blowup of the array of Fig. 2d.

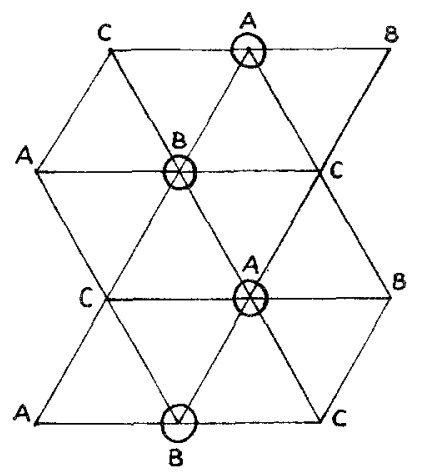

will be critical is that there are the circled vertices interior to the array and that $\frac{1}{2} L l$ (modulo end effects) triangles have two interior vertices.

With these lengthy preliminaries out of the way, we can now describe the Schick-Griffiths model. Each spin takes three possible values, say $a, b, c$. As usual, $P_{\alpha}(a)$ is the projection onto state $a$ at site $\alpha$. Then the basic Hamiltonian is

$$
-H=\sum_{i \in \Lambda^{*}}\left(\frac{1}{2} M Q_{i}+\frac{1}{2} K R_{i}\right)
$$

where

$$
\begin{aligned}
Q_{i} & =\sum_{x=a, b, c} P_{A_{i}}(x) P_{B_{i}}(x) P_{C_{i}}(x) \\
R_{i} & =\sum_{x \neq y} P_{A_{i}}(x) P_{B_{i}}(x) P_{C_{i}}(y)+P_{A_{i}}(x) P_{B_{i}}(y) P_{C_{i}}(x)+P_{A_{i}}(y) P_{B_{i}}(x) P_{C_{i}}(x)
\end{aligned}
$$

That is, of the 27 possible configurations for a triangle, the three with all equal spins have energy $-M / 2$, the 18 with two equal spins have energy $-K / 2$, and the six with three unequal spins have energy 0 . It is easy to see that $H$ has the form $A+\theta A$ for any reflection. We claim:

Theorem 3.7. Consider the Hamiltonian (3.16). Then under our working hypothesis there exists an $N$ so that when $K<-N$ or $K-M<N$, the model has multiple equilibrium states (multiple states invariant and ergodic under those translations leaving the three basic sublattices invariant). If $M>0$, there are at least three, if $M<0$ at least six, and if $M=0$ at least nine (see Fig. 4).

We note that as one increases $K$ with $M$ fixed, one has a situation which is very like that in the antiferromagnet as $h$ is increased, so presumably one does not have a first-order phase transition. Our method of proof shows that 


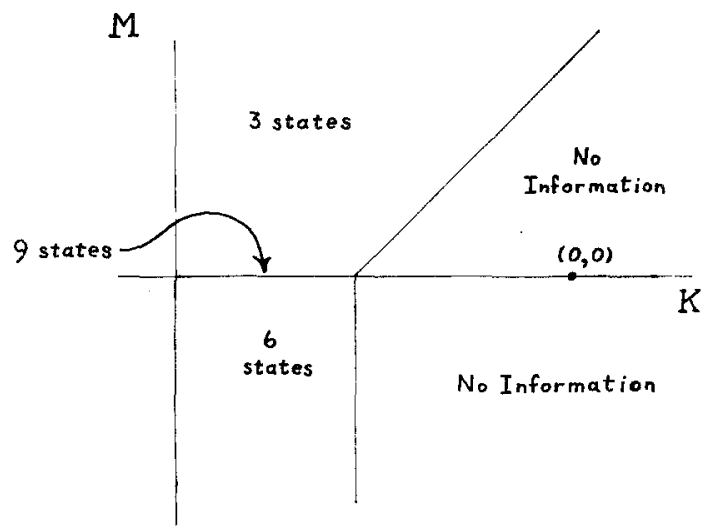

Fig. 4. Phase diagram for the Schick-Griffiths model.

on crossing the line segment $M=0, K<-N$, there is a first-order phase transition: indeed our proof shows that in that region

$$
\varlimsup_{\Lambda \rightarrow \infty}\left\{\left\langle\left(\frac{1}{2|\Lambda|} \sum_{i} Q_{i}\right)^{2}\right\rangle_{\Lambda}-\left\langle\frac{1}{2|\Lambda|} \sum_{i} Q_{i}\right\rangle_{\Lambda}^{2}\right\}>0
$$

whence a first-order transition follows by the Griffiths argument (see Ref. 6).

Proof of Theorem 3.7. Let us label the 27 states for a triangle, using 1-3 for the ferromagnetic states (all spins equal) and 4-9 for the six antiferromagnetic states (all spins different). Let $P_{i}(k), i \in \Lambda^{*}, k \in\{1, \ldots, 27\}$ be the obvious projections. We prove below that

$$
\left\langle P_{i}(k)\right\rangle \leqslant \exp \left[\frac{1}{4} K-\frac{1}{4} \max (M, 0)\right] \quad \text { for } k \geqslant 10
$$

By the obvious symmetries of permuting the labels $a, b, c$ and translation invariance:

$$
\left\langle P_{i}(1)\right\rangle=\left\langle P_{i}(2)\right\rangle=\left\langle P_{i}(3)\right\rangle ; \quad\left\langle P_{i}(4)\right\rangle=\cdots=\left\langle P_{i}(9)\right\rangle
$$

Moreover, when $M=0$ there is a special symmetry ${ }^{(46)}$ : namely leave the $A$-lattice spins alone, permute the $B$-lattice spins $a \rightarrow b \rightarrow c \rightarrow a$ and the $C$-lattice spins $a \rightarrow c \rightarrow b \rightarrow a$. Thus

$$
\left\langle P_{i}(1)\right\rangle=\cdots=\left\langle P_{i}(9)\right\rangle \quad \text { if } M=0
$$

so that $\left\langle P_{i}(1)\right\rangle \leqslant \frac{1}{9}$ for $M=0$. Then, since $\left\langle\sum_{k=1}^{3} P_{i}(k)\right\rangle$ is monotone increasing in $M$ for $K$ fixed,

$$
\left\langle P_{i}(1)\right\rangle \leqslant \frac{1}{9} \quad \text { for } M \leqslant 0
$$


Similarly, $\left\langle\sum_{k=4}^{9} P_{i}(k)\right\rangle$ is decreasing in $K$ if $M-K$ is fixed, so

$$
\left\langle P_{i}(4)\right\rangle \leqslant \frac{1}{9} \quad \text { for } M \geqslant 0
$$

Pick $N$ so that $18 \exp \left(\frac{1}{4} N\right)<\frac{1}{6}$. Then by (3.17)-(3.20)

$$
\begin{array}{ll}
\left\langle P_{i}(1)\right\rangle=\ldots=\left\langle P_{i}(9)\right\rangle>5 / 54 & \text { if } M=0, \quad K<-N \\
\left\langle P_{i}(1)\right\rangle=\left\langle P_{i}(2)\right\rangle=\left\langle P_{i}(3)\right\rangle>1 / 18 & \text { if } M \geqslant 0, \quad K<-N+M \\
\left\langle P_{i}(4)\right\rangle=\cdots=\left\langle P_{i}(9)\right\rangle>1 / 12 & \text { if } M \leqslant 0, \quad K<-N
\end{array}
$$

From these facts the theorem is proven if we show that (3.17) holds and for some small (calculable) $\epsilon$

$$
\left\langle P_{i}(k) P_{j}(l)\right\rangle \leqslant \epsilon \quad \text { all } i, j ; \quad k \neq l, \quad k, l \in\{1, \ldots, 9\}
$$

so long as $N$ is large and $K-\max (M, 0)<N$. Notice that two triangles in different states from 1 to 9 cannot have an edge in common. Thus if $P_{i}(k) P_{j}(l)=1$, there is a chain of triangles in states $10-27$ connected vertex-tovertex and separating $i$ and $j$, as do the shaded triangles in Fig. 5. Such chains (of triangles, rather than line segments) will be considered as "contours." In the usual way, ${ }^{111,13)}(3.21)$ is proven if we show that the probability of a contour $\gamma$ is dominated by $q^{|\gamma|}$ with $q \rightarrow 0$ as $N \rightarrow \infty$. We are thus reduced to proving

$$
\left\langle\prod_{|\gamma| i^{\prime} s} P_{i}(k(i))\right\rangle \leqslant \exp \left\{|\gamma|\left[\frac{1}{4} K-\frac{1}{4} \max (M, 0)\right]\right\}
$$

for all $k(i) \geqslant 10$. Notice that (3.22) includes the missing estimate (3.17) as a special case. To prove (3.22), we proceed as indicated in the general discussion above until we reach arrays of the type in Fig. 3. The expectation of such an array is the sum of all configurations consistent with the configurations in the array determined by $\prod_{i} P_{i}(k(i))$ divided by the sum of all configurations. For each term in the numerator consider the term in the denominator determined by the following rules: If $M \geqslant 0$, change the spins at the interior $A$ and $B$

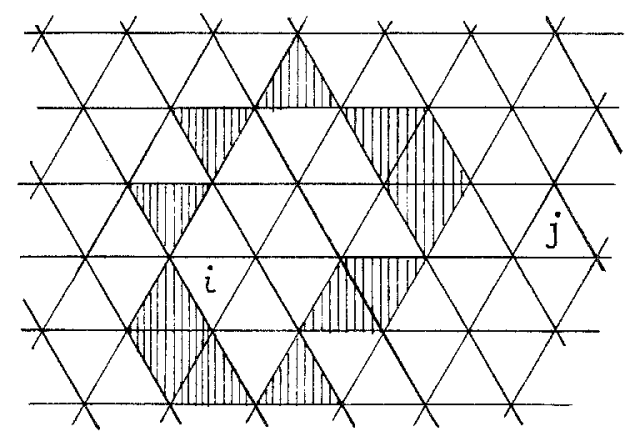

Fig. 5. A contour separating $i$ and $j$. 


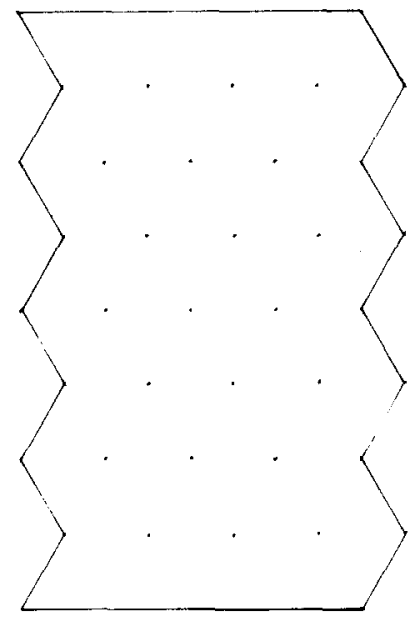

Fig. 6. A special boundary condition.

sites to agree with that on the $C$ sites (which must agree by the method which led to the array). If $M<0$, change the spins at the interior $A$ and $B$ sites so that the interior $A, B, C$ triangles have one of each spin. By this procedure the energy is changed by at least $\frac{1}{4} \max (M, 0)-\frac{1}{4} K$ times the number of interior triangles. Since half the triangles are interior, we have proven (3.23).

Remarks. 1. The Pirogov-Sinai method ${ }^{(43,44)}$ shows that the theorem holds without any hypothesis on RP for $\langle\cdots\rangle_{\infty}$.

2. We illustrate our remark on hexagonal lattices in the Schick-Griffiths model. Consider a model on the hexagonal lattice with three states and pair interactions between nearest neighbors with the following energies: $M$ for $b b ; K$ for $a b, b c, a a, c c$; and 0 for $a c$. This is just the Schick-Griffiths Hamiltonian with an additional interaction forcing the spins at $B$ sites to be in state $b$. The analysis above still works; the net result is an analog of Theorem 3.7 with three, six, or nine states replaced by one, two, or three states.

3. We wish to remark that so long as there is a unique equilibrium state which is translation invariant and invariant under permuting all $a, b, c$ labels, then $\langle\cdots\rangle_{\infty}$ will be RP. Construct an infinite-volume state by taking a region. like that in Fig. 6 with periodic boundary conditions. The infinite-volume state will be RP about horizontal planes with all the symmetries guaranteeing uniqueness. Thus if our working hypothesis fails there must be multiple equilibrium states with all the above symmetries.

\section{HARD-CORE LATTICE GASES}

By a hard-core lattice gas, we mean the system on a regular array with configurations of "occupied" sites. Two sites with $|\alpha-\beta| \leqslant d$ cannot both be occupied, and the statistical weight of a configuration with $N$ sites occupied 
is $z^{N}$. The only parameter of the theory is $z$. The first basic question concerns whether there are multiple equilibrium states for large $z$.

Model 4.1. (Nearest-neighbor, hard-core square lattice gas.) We work on a unit square lattice and take $d=1$. We will recover the result of Dobru$\operatorname{shin}^{(5)}$ that there are multiple equilibrium states if $z$ is large.

Let $\sigma_{\alpha}=+1(-1)$ correspond to site $\alpha$ occupied (resp. unoccupied). We first claim that the interaction is RP about any lattice line. Since the statistical weight is just

$$
\lim _{J \rightarrow \infty} \exp \left[-\sum_{\langle\alpha \beta\rangle} J\left(\sigma_{\alpha}+1\right)\left(\sigma_{\beta}+1\right)+\ln z \sum \frac{1}{2}\left(\sigma_{\alpha}+1\right)\right]
$$

this follows by our general analysis of interactions which are finite. By Theorem 4.3 of Ref. 11, for nearest neighbors $\alpha, \beta$ in a $2 L \times 2 L$ lattice

$$
\begin{aligned}
\left\langle P_{\alpha}(-) P_{\beta}(-)\right\rangle & \leqslant \sum_{\substack{s= \pm 1 \\
t= \pm 1}}\left\langle P_{\alpha}(-) P_{\beta}(-) P_{\gamma}(s) P_{\delta}(t)\right\rangle \\
& \leqslant\left(1 / z^{|\Lambda| / 2}\right)^{1 /(2|\Lambda|)}+2\left(z^{|\Lambda| / 4} / z^{|\Lambda| / 2}\right)^{1 /(2|\Lambda|)} \\
& \leqslant 3 \max \left(z^{-1 / 4}, z^{-1 / 8}\right)
\end{aligned}
$$

where $\alpha, \beta, \gamma, \delta$ form a square.

Since $\left\langle P_{\alpha \alpha}(+)\right\rangle=\left\langle P_{\beta}(+)\right\rangle$ and $\left\langle P_{\alpha}(+) P_{\beta}(+)\right\rangle=0$, we see that

$$
\left\langle P_{\alpha}(+)\right\rangle \geqslant \frac{1}{2}\left(1-3 z^{-1 / 8}\right) \quad \text { for } z>1
$$

Next we claim that for $\alpha, \gamma$ in different sublattices

$$
\left\langle P_{\alpha}(+) P_{\gamma}(+)\right\rangle \leqslant \epsilon(z)
$$

with $\epsilon \rightarrow 0$ as $z \rightarrow \infty$. This and (4.1) imply absence of mixing and existence of multiple equilibrium states. To prove (4.2) we let $\beta$ be a neighbor of $\gamma$ and note that $P_{\gamma}(+) \leqslant P_{\beta}(-)$. Thus (4.2) follows from

$$
\left\langle P_{\alpha}(+) P_{\beta}(-)\right\rangle \leqslant \epsilon(z)
$$

for $\alpha, \beta$ in the same sublattice, say $A$. [Notice (4.3) is a direct statement of long-range order]. To prove (4.3), given any configuration we fill in the squares formed by joining together the nearest neighbors of each occupied $A$-lattice site. (These squares have sides which are diagonals of the basic lattice unit square.) The contours are connected components of the boundary of the resulting filled-in region. As usual, (4.3) follows from

$$
\text { (probability of contour } \gamma \text { ) } \leqslant[\delta(z)]^{|\gamma|}
$$

with $\delta \rightarrow 0$ as $z \rightarrow \infty$. We will prove (4.4a) with

$$
\delta(z)=z^{-1 / 4}
$$


To prove (4.4), let $l$ be a line in $\gamma$. Since $l$ is on a boundary, the square $\Delta$ containing $l$ has one occupied and three vacant sites. Thus if $P_{l}$ is the projection onto this configuration

$$
\text { (probability of } \gamma) \leqslant\left\langle\prod_{l \in \gamma} P_{l}\right\rangle \leqslant\langle Q\rangle^{|\gamma||| \Lambda \mid}
$$

where $Q$ is the universal projection obtained by using Theorem 4.3 of Ref. 11 , i.e., $Q$ has every lattice site with both coordinates even occupied, and all other sites vacant. Thus

$$
\langle Q\rangle \leqslant z^{|\Lambda| / 4} / z^{|\Lambda| / 2}
$$

proving (4.4).

Model 4.2. (Nearest-neighbor, hard-core triangular lattice gas.) We now work on the unit triangular lattice with $d=1$. As in Model 3.5, we suppose that $\langle\cdots\rangle_{\infty}$ is RP. By the analysis of Model 3.5 and the fact that in an array of Fig. 3 with all sites empty we can gain a factor of $z^{\frac{t}{t} \text { triangles/8 }}$ by filling in the interior $A$ sites (there is one such site for every eight triangles), we have for $\alpha, \beta, \gamma$ forming a triangle

$$
\left\langle P_{\alpha}(-) P_{\beta}(-) P_{\gamma}(-)\right\rangle \leqslant z^{-1 / 8}
$$

so that

$$
\left\langle P_{\alpha}(+)\right\rangle \geqslant \frac{1}{3}\left(1-z^{-1 / 8}\right)
$$

As in Model 4.1, we need only show that for $\alpha, \beta$ in the $A$ sublattice

$$
\left\langle P_{\alpha}(+) P_{\beta}(-)\right\rangle \leqslant \epsilon(z)
$$

with $\epsilon \rightarrow 0$ as $z \rightarrow \infty$. Given a configuration, we fill in the hexagons formed by all triangles with an occupied $A$ vertex. Contours are again connected components of boundary. Each edge $J$ of a contour is associated to a unique triangle $i(J)$ with $\sigma_{A_{i(J)}}=-1$. Thus

$$
\begin{aligned}
\text { (probability of contour } \gamma) & \leqslant\left\langle\prod_{J \in \gamma} P_{A_{i(J)}}(-) P_{B_{i(J)}}(-) P_{C_{i(J)}}(-)\right\rangle \\
& \leqslant\left(z^{-1 / 8}\right)^{|\gamma|}
\end{aligned}
$$

as above. Modulo the hypothesis on $\langle\cdots\rangle_{\infty}$ being RP, we have proven the existence of at least three equilibrium states, a result of Heilmann. ${ }^{(25)}$ (Without that assumption, we can only conclude that there are at least two states.)

Model 4.3. (Nearest-neighbor, hard-core hexagonal lattice gas.) We now work on the unit hexagonal lattice with $d=1$. Thinking of this as the triangular lattice with $\sigma_{\alpha}=-1$ for $\alpha$ in the $B$ lattice, we can follow the above analysis directly. Contours are defined as in Model 4.2 and

$$
\text { (probability of } \gamma \text { ) } \leqslant z^{-1 / 8|\gamma|}
$$


still holds. The only difference is that $\left\langle P_{\alpha}(+)\right\rangle \geqslant \frac{1}{2}\left(1-z^{-1 / 8}\right)$ for $\alpha$ in the $A$ or $C$ sublattice. We have at least two states, a result of Heilmann. ${ }^{(25)}$

We summarize the last three models:

Theorem 4.1. The hard-core, nearest-neighbor lattice gas on the square, triangular, and hexagonal lattices have at least two equilibrium. states for large $z$, and, for the square lattice, long-range order. If $\langle\cdots\rangle_{\infty}$ is $\mathrm{RP}$ in the triangular and hexagonal lattices, there is long-range order in those cases and at least three states for the triangular lattice.

Model 4.4. (Next-nearest-neighbor, hard-core square lattice gas.) This is the model on the square lattice with $d=\sqrt{2}$. It is still RP with respect to reflection in lattice lines, but not with respect to diagonals. The basic chessboard estimate is thus Theorem 4.3 of Ref. 11. One can still attempt to define contours with the basic "blob" being a square with side 2 and sides parallel to the original lattice. However, one can no longer identify the necessary four-spin blocks of minus around a contour; indeed, one can see that only corners of contours count. This fact suggests that the analog of this model in three or more dimensions has multiple equilibrium states. In two dimensions, one can easily find an infinity of states of finite energy breaking the longrange order. This suggests no multiple states, which is the conventional wisdom.

Model 4.5. This is the model on the square lattice which results if one thinks of an occupied site as being the shape shown in Fig. 7. Thus pairs with $d=1, \sqrt{2}, 2, \sqrt{5}, 3$, and $\sqrt{10}$ are not allowed but $d=2 \sqrt{ } 2$ is. One can develop a contour analysis about sites $(n, m)$ with $n+m \equiv 0 \bmod 4$ and thereby see that there are four ground states and the Peierls condition of Pirogov and Sinai ${ }^{(42-44)}$ holds. By their theory, one gets four states at large $z$.

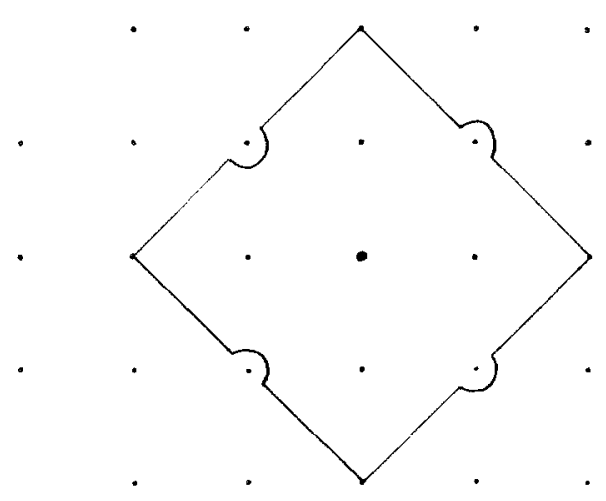

Fig. 7. Hard core for Model 4.5. 
Our method yields no information since the model has no RP. We see once again the limitations of RP methods!

Model 4.6. (Widom-Rowlinson model.) The basic lattice is the square lattice. At each site there are three possible states $A, B$, or 0 (=vacant) with the constraint that one cannot have $A$ and $B$ at nearest neighbor sites. The weight of a configuration is $z^{m}$ with $m$ the number of sites with $A$ or $B$. The applicability of naive contour arguments is due to Gallavotti and Lebowitz, ${ }^{(20)}$ with recent simplifications by Bricmont et ll $^{(3)}$ With RP methods it is effortless to prove:

Theorem 4.2. The Widom-Rowlinson lattice model has multiple phases for $z$ large, and long-range order with $P_{\alpha}(A)-P_{\alpha}(B)$ as order parameter.

Proof. RP in lines containing lattice sites and bonds holds as in Model 4.1. By considering the 17 possibilities for filling in a square with one corner vacant, one sees for $z>1$,

$$
\left\langle P_{\alpha}(0)\right\rangle \leqslant 17 z^{-1 / 4}
$$

so by symmetry $P_{\alpha}(A)=P_{\alpha}(B) \rightarrow 1 / 2$ as $z \rightarrow \infty$. Thus we only need to show that $\left\langle P_{\alpha}(A) P_{\gamma}(B)\right\rangle \rightarrow 0$ as $z \rightarrow \infty$. Draw a contour of conventional type between dual lattice sites by filling in a square about every vacant site. If $\alpha$ and $\gamma$ are occupied by different species, a contour must separate them. For every dual lattice site on a contour, the corresponding square has at least one vacant and one occupied site. Thus (again counting possibilities)

$$
\text { (probability of } \gamma \text { ) } \leqslant\left(5 z^{-1 / 4}\right)^{|\gamma|}
$$

Remarks. 1. The above proof works if there is also next-nearest-neighbor $A B$ exclusion, but longer range exclusion destroys RP!

2. Unlike the naive approach, ${ }^{(3,20)}$ we do not need $A-B$ symmetry for the contour argument. For example, if in addition to the $A B$ exclusion one has a Hamiltonian

$$
J \sum_{\langle\alpha \beta\rangle} P_{\alpha}(A) P_{\beta}(A)-\mu_{A} \sum P_{\alpha}(A)-\mu_{B} \sum P_{\alpha}(B)
$$

one can easily show that for $J, \mu_{A}$ fixed and $\beta$ large there is a $\mu_{B}(\beta) \rightarrow \mu_{A}+2 J$ as $\beta \rightarrow \infty$ at which two phases occur. As usual, the Pirogov-Sinai method also yields these results.

\section{COULOMB LATTICE GASES}

Thus far, there have been a number of places where we emphasized the limitations of RP methods. In this section, we discuss examples which illustrate the opposite side of the coin. Indeed, the results in this section are 
all new and we do not know how to obtain them with any other methods, for all other methods require contours whose width is the range of the interaction, which is infinite here. In contrast, we will use RP methods and a width-two contour.

In all the models, we will suppose that our lattice is $\mathbb{Z}^{v}$ with $\nu=3$. It is easy to extend the method to $\nu \geqslant 3$. One can treat the classical case of $\nu=2$ by replacing the Coulomb potential by a Yukawa potential of mass $\mu$ and then taking $\mu \rightarrow 0$. The sole effect is to suppress all nonneutral configurations. In all the models, it is easy to check stability of the energy essentially because the point charges of a lattice Coulomb gas can be replaced by spheres with finite self-energy. For a proof that a sensible thermodynamics exists, see Refs. 14 and 15.

Model 5.1. (Coulomb monopole, plus, minus charges.) At each site we have a charge $\sigma_{\alpha}= \pm 1$ and the Hamiltonian is $H=\sum_{\alpha \neq \beta} \alpha_{\alpha} \sigma_{\beta}|\alpha-\beta|^{-1}$. In a periodic boundary condition box, there is some care needed: only neutral configurations have finite energy, and $H^{\text {per }}(\sigma)$ is defined by extending the configuration in $\Lambda$ periodically and letting

Alternatively

$$
H^{\text {per }}(\sigma)=\lim _{L \rightarrow \infty} \frac{1}{L^{3}} \sum_{\substack{\alpha, \beta \in L \Lambda \\ \alpha \neq \beta}} \sigma_{\alpha} \sigma_{\beta}|\alpha-\beta|^{-1}
$$

$$
H^{\mathrm{per}}(\sigma)=\sum_{p \in \Lambda^{*}} \hat{\sigma}_{p} \hat{\sigma}_{-p} E_{p}
$$

where as usual $\hat{\sigma}_{p}=|\Lambda|^{-1 / 2} \sum e^{i \alpha \cdot p} \sigma_{\alpha}$ and $E_{p}$ is $(2 \pi)^{3 / 2}$ times the Fourier transform of $\sum_{\alpha \neq 0}|\alpha|^{-1} \delta(x-\alpha)$. The $E_{p}$ can be evaluated by the Poisson summation formula as $\sum_{\beta} f(p+2 \pi \beta)$, where $f(p)=p^{-2}-\int(p-l)^{-2} \hat{\chi}(l) d^{3} l$, with $\hat{\chi}$ a function whose Fourier transform vanishes for $|x|>1$ and $\int \hat{\chi}(l) d^{3} l=1$. Notice that $f$ falls off faster than any power, so the sum over $\beta$ is convergent.

We claim next that this model is RP under a $\theta$ obtained by reflecting in a plane and changing the sign of the charge. By the basic machinery of Section 3 of Ref. 11, this follows if we show that for any charge configuration $r$ on the right side of the plane $\alpha_{1}=1 / 2$ and the configuration $l$ obtained by reflecting and sign shift we have that

$$
-\sum_{\substack{\alpha 1>\frac{1}{3} \\ \beta_{1}<\frac{1}{2}}} \sigma_{\alpha} \sigma_{\beta}|\alpha-\beta|^{-1} \geqslant 0
$$

This, in turn, follows if we prove the stronger result

$$
\int_{\substack{x_{1}>0 \\ y_{1}<0}} \rho(x) \rho(y)|x-y|^{-1} \geqslant 0
$$

if $\rho\left(-x_{1}, x_{2}, x_{3}\right)=\rho\left(x_{1}, x_{2}, x_{3}\right)$. The result (5.2) is well known (see, e.g., Section 5 of Ref. 11). 
Remark. The nearest neighbor antiferromagnet has the same RP, so one can ask if the present model is RP under reflection in lattice planes. The answer is no. This can be seen as follows: if it were, one would have the chessboard estimate, Theorem 4.3 of Ref. 11, so that the probability $p$ of eight neighboring plus charges would be dominated by $q=\langle Q\rangle^{1 /[\Lambda \mid}$, where $Q$ assigns a plus charge to each site. But $q=0$ by the requirement of overall neutrality, while clearly $p>0$.

To find the ground states, we need only consider configurations which alternate signs in two directions and are a two-unit reflecting sequence in the third direction, i.e., $x y$ means $\cdots x y-y-x x y-y-x \cdots$. The two possibilities are given in the first column of Table III with $\mu$, a parameter of Model 5.2, set to zero. In this case $a=1.74756459, b=1.6170762, c=$ 0.84116805 ( $c$ is irrelevant in this model). $a$ is just the constant computed by Madelung in his famous paper. ${ }^{(38)}$ We have evaluated $b$ and $c$ using Hund's method $^{(29)}$ and the tables in Ref. 45. For additional discussion of Madelung constants, see Refs. 31,52 , and 54-57. For the case at hand, all that matters is that $a>b$. We will show that this implies:

Theorem 5.1. The $\sigma_{\alpha}= \pm 1$ Coulomb monopole gas has at least two equilibrium states at large $\beta$.

Proof. As usual let $P_{\alpha}( \pm)$ be the projection onto $\sigma_{\alpha}= \pm 1$. Clearly, by symmetry $\left\langle P_{\alpha}(+)\right\rangle=\left\langle P_{\alpha}(-)\right\rangle=1 / 2$. Thus we only need to show that

$$
\sup _{\alpha, \delta}\left\langle P_{\alpha}(+) P_{\delta}\left(-(-1)^{|\alpha-\delta|}\right)\right\rangle \rightarrow 0
$$

as $\beta \rightarrow \infty$, i.e., there is long-range order favoring equal spins on the $A$ sublattice and the opposite spins on the $B$ sublattice. Draw contours by placing square faces between neighboring spins of the same sign. If the contour $\gamma$ has $|\gamma|$ squares, then the probability of $\gamma$ is dominated by a product of $|\gamma|$ projections $P_{i}$, each one the projection onto two neighboring equal spins. There are three directions in which the axis between the neighbors may point and two possibilities for whether the $A$ site is to the "left" ("above" or ...) of the $B$ site. By picking the combination that occurs most often, we obtain a bound on the probability of $\gamma$ which has at least $|\gamma| / 6$ projections, all of the same type, say $A$ on the left, $B$ on the right. Using a chessboard estimate with

Table III

\begin{tabular}{ccc|ccc}
\hline$n$ & Block & $-e(n)$ & $n$ & Block & $-e(n)$ \\
\hline 1 & +- & $a+\mu$ & 3 & +0 & $c+\frac{1}{2} \mu$ \\
2 & ++ & $b+\mu$ & 4 & 00 & 0 \\
\hline
\end{tabular}


a single unit in two directions and a double unit in the third, we get prob $(\gamma) \leqslant$ $\alpha^{|\gamma|}$, where $\alpha=\exp \left[\frac{1}{6} \beta(b-a)\right]$, which implies the result.

Model 5.2. (Coulomb monopole gas;,,+- 0 charges.) The model is the same as in Model 5.1, except that now $\sigma_{\alpha}$ can take the values \pm 1 or 0 . Now $H$ is $\sum_{\alpha, \beta ; \alpha \neq \beta} \sigma_{\alpha} \sigma_{\beta}|\alpha-\beta|^{-1}-\mu \sum\left|\sigma_{\alpha}\right|$, so with $z=e^{\beta \mu}$ we have a Coulomb gas with fugacity $z$. Now the relevant energies are given by the four entries in Table III. For $\mu$ large, indeed for $\mu>-a$, we have two phases at low temperatures essentially by the argument in Model 5.1 supplemented by the fact that

$$
\left\langle P_{\alpha}(0)\right\rangle \leqslant \exp [-\beta(a+\mu)]
$$

by a chessboard estimate. An interesting feature takes place near $\mu \sim-a$. Notice that $2 c<a$, so that for $\mu \sim-a, c+\frac{1}{2} \mu<a+\mu$. By a contour argument of the type in Model 5.1, we can make

$$
\left\langle P_{\alpha}(+)\left[P_{\gamma}(0)+P_{\gamma}\left(-(-1)^{|\alpha-\gamma|}\right)\right]\right\rangle
$$

small so long as $\exp \{-\beta[e(2)-e(1)]\}$ and $\exp \{-\beta[e(3)-e(1)]\}$ are both small. This will hold uniformly for $\mu \in(-a-\delta,-a+\delta)(\delta$ small) if $\beta$ is large. By the strategy of Section 1.3, we find $\mu(\beta) \rightarrow-a$ as $\beta \rightarrow \infty$, so that there are three phases at fugacity $z=e^{\beta \mu(\beta)}$. Two phases are like those of Model 5.1 and can be thought of as "crystal" phases. One has mainly empty sites and can be thought of as a "plasma." We summarize with:

Theorem 5.2. The Coulomb monopole gas with $\sigma_{\alpha}= \pm 1,0$ has at least three phases for $\beta$ large and suitable fugacity $z(\beta)$.

We are indebted to David Brydges for having checked that his proof of Debye screening ${ }^{(4)}$ also applies to the lattice Coulomb gas, provided the activity is sufficiently small and the temperature is sufficiently large. Thus, we conclude that the lattice Coulomb gas has a single-phase region with exponential clustering apart from the two- and three-phase regions exhibited in this paper.

Model 5.3. (Monopole gas with a lattice Coulomb potential.) In the last two examples, it appeared that numerical relations among Madelung constants played a significant role. We want to show that this is no accident by considering a more general model. A special case of particular interest of this more general model is the lattice Coulomb potential,

$$
V(\alpha-\beta)=\frac{1}{(2 \pi)^{3}} \int_{\left|p_{\mathfrak{i}}\right| \leqslant \pi}\left(3-\cos p_{1}-\cos p_{2}-\cos p_{3}\right)^{-1} e^{i p \cdot(\alpha-\beta)} d^{3} p
$$


Other examples would include the lattice Yukawa potential [replace 3 in. (5.3) by $3+\mu^{2}$ ] and the continuum Yukawa potential restricted to lattice sites. Our Hamiltonian is now

$$
H=\sum_{\alpha, \beta} V(\alpha-\beta) \sigma_{\alpha} \sigma_{\beta}-\mu \sum_{\alpha}\left|\sigma_{\alpha}\right|
$$

where $V$ is assumed RP, i.e.,

$$
\sum_{\substack{\alpha 1 \geqslant 1 \\ \beta_{1} \geqslant 1}} \bar{z}_{\alpha} z_{\beta} V\left(\alpha_{1}+\beta_{1}-1, \alpha_{2}-\beta_{2}, \alpha_{3}-\beta_{3}\right) \geqslant 0
$$

For simplicity, we also suppose that $V\left(\alpha_{1}, \alpha_{2}, \alpha_{3}\right)$ is symmetric under permutations of $\alpha_{1}, \alpha_{2}, \alpha_{3}$. As in Model 5.1, we have reflection positivity under $\sigma_{\alpha} \rightarrow-\sigma_{r \alpha}$. By general principles, ${ }^{(11)}$ the periodic state is RP also. Let $\hat{V}$ be the Fourier transform of $V$ normalized by

$$
V(\alpha)=\frac{1}{(2 \pi)^{3}} \int_{\left|p_{i}\right| \leqslant \pi} \hat{V}(p) e^{i p \cdot \alpha} d^{3} p
$$

In finite volume $\Lambda$, one finds that (see Ref. 11)

$$
H_{\Lambda}(\sigma)=\sum_{p \in \Lambda^{*}} \hat{V}(p) \hat{\sigma}_{p} \hat{\sigma}_{-p}
$$

where $\hat{\sigma}_{p}=|\Lambda|^{-1 / 2} \sum_{\alpha \in \Lambda} e^{i p \cdot \sigma_{\alpha}}$ and $\Lambda^{*}$ is the dual set of $p$ 's to $\alpha \in \Lambda$. If we consider the configuration corresponding to state 1 of Table III, i.e., $\sigma_{\alpha}=$ $(-1)^{\alpha_{1}+\alpha_{2}+\alpha_{3}}$, we find that $\hat{\sigma}_{p}=|\Lambda|^{1 / 2}$ if each $p_{i}= \pm \pi$ and 0 otherwise. Thus

$$
a=-\hat{V}(\pi, \pi, \pi)
$$

[In the sum over $\Lambda^{*}$ only one point of $( \pm \pi, \pm \pi, \pm \pi)$ enters, i.e., these are equivalent $p$ 's, and in particular $\hat{V}( \pm \pi, \pm \pi, \pm \pi)=\hat{V}(\pi, \pi, \pi)]$. For the configuration corresponding to state 2 , we find that $\hat{\sigma}_{p}=\frac{1}{2}|\Lambda|^{1 / 2}(1 \mp i)$ at $p=(\pi, \pm \pi / 2, \pi)$ and zero at all nonequivalent points. Thus, noting that $\hat{V}(-\pi / 2, \pi, \pi)=\hat{V}(\pi / 2,-\pi,-\pi)$ (by reality of $V)=\hat{V}(\pi / 2, \pi, \pi)$ (since $\pi$ is equivalent to $-\pi)$, we see that

$$
b=-V(\pi / 2, \pi, \pi)
$$

For the configuration corresponding to state 3 , we have $\hat{\sigma}_{p}=\frac{1}{4}|\Lambda|^{1 / 2}(1 \pm i)$ for $p=( \pm \pi / 2, \pi, \pi), \frac{1}{2}|\Lambda|^{1 / 2}$ for $p=(\pi, \pi, \pi)$ and zero for nonequivalent points. Thus

$$
c=-\frac{1}{4} \hat{V}(\pi, \pi, \pi)-\frac{1}{4} \hat{V}(\pi / 2, \pi, \pi)
$$

We see that $a, b, c$ are not independent, since

$$
4 c=a+b
$$


which can be checked for the Madelung constants! In particular, the two critical inequalities

$$
a>b, \quad a>2 c
$$

are equivalent to the single inequality

$$
\hat{V}(\pi, \pi, \pi)<\hat{V}(\pi / 2, \pi, \pi)
$$

We claim that (5.5) implies (5.10) so long as $V \rightarrow 0$ at infinity and $V \not \equiv 0$. For, let $\tilde{V}(n)$ be the Fourier transform of $V(n, m, l)$ in the $m, l$ variables evaluated at $p_{2}=p_{3}=\pi$. Then (5.5) implies that $\tilde{V}(n)$ is RP as a one-variable object. Thus, by the general theory ${ }^{(11)}$

$$
\widetilde{V}(n)=\int_{-1}^{1} d \rho(\lambda) \lambda^{|n|-1}, \quad|n| \geqslant 1
$$

for some positive measure $d \rho$. It follows that (up to a constant which can be absorbed into the definition of $\tilde{V}$ )

$$
\tilde{V}\left(p_{1}, \pi, \pi\right)=c_{0}+\int_{-1}^{1} 2 d \rho(\lambda)\left(\cos p_{1}-\lambda\right) /\left(1+\lambda^{2}-2 \lambda \cos p_{1}\right)
$$

so that (5.10) follows from

$$
-(\lambda+1)^{-1}<-\lambda /\left(\lambda^{2}+1\right), \quad \text { for }|\lambda|<1
$$

and the fact that $d \rho$ cannot be concentrated entirely at \pm 1 , since $\tilde{V}(n) \rightarrow 0$ at $\infty$. We have thus proven:

Theorem 5.3. The conclusions of Theorems 5.1 and 5.2 remain true if the Coulomb potential $|\alpha-\beta|^{-1}$ is replaced by $V(\alpha-\beta)$ for any $V$ obeying (5.5) and not identically zero.

Alternatively, inequalities (5.9) with $>$ replaced by $\geqq$ follow directly from the chessboard estimates (e.g., the probability of configuration 3 of Table III in some finite volume is dominated by the geometric mean of 1 and 4 , so $c \geqq \frac{1}{2} a$ ). In fact, if we check the conditions required for the CauchySchwarz inequalities used to obtain chessboard estimates to be equalities, we find that this would require $V \equiv 0$.

Finally, we should prove that the lattice Coulomb potential obeys (5.5). Letting $\widetilde{V}\left(n, p_{2}, p_{3}\right)$ be the partial Fourier transform of $V$, we see that (5.5) is equivalent to $\widetilde{V}\left(n, p_{2}, p_{3}\right)$ being RP in $n$ for each fixed $p_{2}, p_{3}$, since

$$
\text { LHS of }(5.5)=\sum_{\substack{\alpha_{1} \geqslant 1 \\ \beta_{1} \geqslant 1}} \int \bar{w}_{\alpha_{1} p_{2} p_{3}} w_{\beta_{1} p_{2} p_{3}} \tilde{V}\left(\alpha_{1}+\beta_{1}-1, p_{2}, p_{3}\right) d p_{2} d p_{3}
$$

with

$$
w_{\alpha_{1} p_{2} p_{3}}=\text { const } \sum_{\alpha_{2} \alpha_{3}} z_{\alpha} \exp \left[i\left(p_{2} \alpha_{2}+p_{3} \alpha_{3}\right)\right]
$$


Thus, by the one-dimensional theory, ${ }^{(11)}(5.5)$ holds if and only if

$$
\tilde{V}\left(p_{1}, p_{2}, p_{3}\right)=c\left(p_{2}, p_{3}\right)+2 \int_{-1}^{1} d \rho\left(\lambda, p_{2}, p_{3}\right)\left(\cos p_{1}-\lambda\right) /\left(1+\lambda^{2}-2 \lambda \cos p_{1}\right)
$$

Letting $\lambda_{0}\left(p_{2}, p_{3}\right)$ be defined by $\frac{1}{2}\left(\lambda_{0}+\lambda_{0}^{-1}\right)=2-\cos p_{2}-\cos p_{3}$ with $0<\lambda_{0}<1$, and choosing

$$
d \rho\left(\lambda, p_{2}, p_{3}\right)=\left[2 \lambda_{0} /\left(1-\lambda_{0}{ }^{2}\right)\right] \delta\left(\lambda-\lambda_{0}\right), \quad c=2 /\left(1-\lambda_{0}{ }^{2}\right)
$$

we find that $\tilde{V}\left(p_{1}, p_{2}, p_{3}\right)=\left(3-\cos p_{1}-\cos p_{2}-\cos p_{3}\right)^{-1}$ has the form of (5.11), verifying that the lattice Coulomb potential is RP. Our choice of $c$ and $d \rho$ will seem less mysterious if one notes first that

$$
2\left(\cos p_{1}-\lambda\right)\left(1+\lambda^{2}-2 \lambda \cos p_{1}\right)^{-1}=-\lambda^{-1}+\left(1+\lambda^{2}-2 \lambda \cos p_{1}\right)^{-1}\left(1-\lambda^{2}\right)
$$

Model 5.4. Consider a Coulomb lattice gas as above, but allow also charges of, say, \pm 2 , with a different chemical potential ( $\mu_{1}$ for \pm 1 charges and $\mu_{2}$ for \pm 2 ). By the same techniques used above, plus the strategy of Section 1.6 , we find that for sufficiently large $\beta$ and some $\mu_{1}(\beta) \simeq-a$ and $\mu_{2}(\beta) \simeq$ $-4 a$ there are at least five phases: two "crystals" of mainly \pm 1 charges, two more "crystals" of mainly \pm 2 , and a "plasma."

Model 5.5. (Dipole gas.) We wish to note that Fröhlich and Spencer ${ }^{(19)}$ have used methods related to those above to prove phase transitions in a model of lattice dipoles where each dipole is required to lie in one of the four positions pointing toward a nearest neighbor.

Model 5.6. (Quantum monopole gas; following Fröhlich.) ${ }^{(10)}$ We consider a quantum model where one can place either of two fermions at each lattice site, one with charge +1 and one with charge -1 . We will show that at suitable temperatures and "fugacities," crystals form, i.e., there is longrange correlation for having opposite charges on the even and odd sublattices. For simplicity, we work in $v \geqslant 3$ dimensions and exploit infrared bounds. In two dimensions with a Yukawa potential, one can prove similar results using the method of "exponential localization." (13) With minor changes, one can accommodate general RP potentials and/or bosons with hard cores (i.e., no more than one particle allowed at each site) in place of fermions; indeed, many of the complications in the fermion case are absent for bosons.

We begin by recalling a few facts about fermion systems with $m<\infty$ degrees of freedom (in our case $m$ will be $2|\Lambda|$ ). There are $m$ operators $\psi_{1}, \ldots, \psi_{m}$ on $\mathbb{C}^{M}\left(M=2^{m}\right)$ obeying

$$
\begin{gathered}
\left\{\psi_{i}, \psi_{j}^{*}\right\}=\delta_{i j} \\
\left\{\psi_{i}, \psi_{j}\right\}=0
\end{gathered}
$$


where $\{A, B\}=A B+B A$ is the anticommutator. All sets on $\mathbb{C}^{M}$ obeying (5.12) are unitarily equivalent. The existence and uniqueness questions for the relations (5.12) are standard; see Ref. 48 or the remarks below. Define

$$
n_{i}=\psi_{i}^{*} \psi_{i}
$$

Then (5.12) implies that

$$
\left[n_{i}, \psi_{j}\right]=0 \quad(i \neq j) ; \quad n_{i} \psi_{i}=\left(n_{i}-1\right) \psi_{i}^{*}=0
$$

Since (5.12a) implies that $\operatorname{Ran} \psi_{i}+\operatorname{Ran} \psi_{i}{ }^{*}=\mathbb{C}^{M}$, we conclude that $n_{i}$ has eigenvalues 0 and 1 only and that $\operatorname{Ran} \psi_{i} \perp \operatorname{Ran} \psi_{i}{ }^{*}$. Two families of unitaries are important: first let

$$
u_{i}=(-1)^{n_{t}}
$$

Then

$$
u_{i} \psi_{j} u_{i}^{-1}=\psi_{j} \quad(j \neq i) ; \quad u_{i} \psi_{i} u_{i}^{-1}=-\psi_{i}
$$

and

$$
u_{i}^{2}=1 ; \quad u_{i} u_{j}=u_{j} u_{i} ; \quad u_{i}^{*}=u_{i}
$$

Second, let

$$
v_{i}=\left(\prod_{j \neq 1} u_{j}\right)\left(\left(\psi_{i}+\psi_{i}^{*}\right)\right.
$$

Then

$$
v_{i} \psi_{j} v_{i}^{-1}=\psi_{j} \quad(j \neq i) ; \quad v_{i} \psi_{i} v_{i}^{-1}=\psi_{i}^{*}
$$

and

$$
v_{i}^{2}=1 ; \quad v_{i} v_{j}=-v_{j} v_{i} \quad(i \neq j) ; \quad v_{i}{ }^{*}=v_{i}
$$

We will need two additional properties of the $\psi_{i}$ :

Proposition 5.4. (a) The $\psi_{i}$ have a simultaneous real matrix representation in which $n_{i}, u_{i}, v_{i}$ are also real.

(b) The $2^{2 m}$ operators $\psi_{1}^{a_{1}(}\left(\psi_{1}{ }^{*}\right)^{b_{1}} \cdots \psi_{m}^{a_{m}}\left(\psi_{m}{ }^{*}\right)^{b_{m}}$, with each $a_{i}$ and $b_{i}$ zero or one, are linearly independent and span $\operatorname{Hom}\left(\mathbb{C}^{M}\right)$. Moreover,

$$
\operatorname{Tr}\left(\psi_{1}^{a_{1}}\left(\psi_{1}^{*}\right)^{b_{1}} \ldots\right)=\prod_{i=1}^{m}\left[\left(1-\frac{1}{2} a_{i}\right) \delta_{a_{i} b_{i}}\right]
$$

Remark. Part (b) implies in particular that any map between two families obeying the CAR (5.12) extends to an automorphism of the corresponding algebras and thus is unitarily implemented since every automorphism of $\operatorname{Hom}\left(\mathbb{C}^{M}\right)$ is so implemented. This yields a proof of uniqueness for the CAR; existence is proven in (a).

Proof. (a) Write $\mathbb{C}^{M}$ as the $m$-fold tensor product $\mathbb{C}^{2} \otimes \cdots \otimes \mathbb{C}^{2}$. Let $n_{i}=1 \otimes \cdots \otimes n \otimes \cdots \otimes 1$ ( $n$ is the ith factor) with $n=\left(\begin{array}{ll}1 & 0 \\ 0 & 0\end{array}\right)$, so that 
$u_{i}=(-1)^{n_{\imath}}=1 \otimes \cdots \otimes u \otimes \cdots \otimes 1$ with $u=\left(\begin{array}{rr}-1 & 0 \\ 0 & 1\end{array}\right)$. Let $p_{i}=1 \otimes \cdots \otimes p$ $\otimes \cdots \otimes 1$ with $p=\left(\begin{array}{cc}0 & 0 \\ 1 & 0\end{array}\right)$. Then $n_{i}=p_{i}{ }^{*} p_{i},\left\{p_{i}, p_{i}{ }^{*}\right\}=1$, and $\left[p_{i}, p_{j}\right]=$ $\left[p_{i}, p_{j}{ }^{*}\right]=0(i \neq j)$. To get the full CAR, we employ a Jordan-Wigner transformation and let

$$
\psi_{i}=\left(\prod_{j<i} u_{j}\right) p_{i}
$$

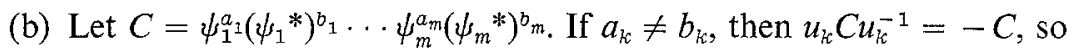
$\operatorname{Tr}(C)=\operatorname{Tr}(-C)=0$. Since the $n_{i}$ commute and $v_{j} n_{i} v_{j}^{-1}=n_{i}(i \neq j)$, $v_{j} n_{j} v_{j}^{-1}=1-n_{j}$, we see that any product of $\left(n_{i}-\frac{1}{2}\right)$ 's has zero trace for the same reason, so that (5.16) holds. Now let $A_{j}=\psi_{j}+\psi_{j}^{*}, B_{j}=i^{-1}\left(\psi_{j}-\psi_{j}^{*}\right)$. Relation (5.16) and the commutation relations among the $A$ 's and $B$ 's imply that the operators $A_{1}^{a_{1}} B_{1}^{b} \ldots$ are orthonormal in the trace inner product $(C, D)=\operatorname{Tr}\left(C^{*} D\right)$. They are thus independent, so that the $\psi_{1}^{a_{1}} \ldots$, which have the same span and as many elements, are also independent. That the span is $\operatorname{Hom}\left(\mathbb{C}^{M}\right)$ follows from counting dimensions.

With these preliminaries out of the way, we can describe the model and analyze it. In finite volume $\Lambda \subset \mathbb{Z}^{\nu}$, we take $m=2|\Lambda|$ and label the $\psi$ 's as $\left\{\psi_{\alpha}{ }^{ \pm}\right\}_{\alpha \in \Lambda}$. We let

$$
\begin{aligned}
q_{\alpha} & =n_{\alpha}^{(+)}-n_{\alpha}^{(-)} \\
T & =-\sum_{\langle\alpha, \beta\rangle \subset \Lambda}\left(\psi_{\alpha}^{*} \psi_{\beta}+\psi_{\beta}^{*} \psi_{\alpha}\right)
\end{aligned}
$$

the sum being over nearest neighbor pairs. We will take in a torus $\Lambda$

$$
H_{\Lambda}=T+\sum_{\alpha, \beta \in \Lambda} V^{(\Lambda)}(\alpha-\beta) q_{\alpha} q_{\beta}-\mu \sum_{\alpha \in \Lambda} q_{\alpha}^{2}
$$

where $V$ is the periodic Coulomb potential and $T$ has periodic boundary conditions. The $T$ in (5.17b) is just the second quantization of $-2 \nu I-\Delta$, where $\Delta$ is the lattice Laplacian. It describes "hopping" between neighboring sites. Often one describes these kinds of models with the sign of $T$ reversed. Since $T$ and $-T$ are unitarily equivalent under $U=\prod_{\alpha \in \Lambda_{\mathrm{even}}} u_{\alpha}{ }^{+} u_{\alpha}{ }^{-}$with $U q_{\alpha} U^{-1}=q_{\alpha}$, the models are equivalent. Our choice of the sign is convenient since it makes $T$ look "ferromagnetic." We aim to prove:

Theorem 5.5. For some $\beta_{c}, \mu_{c}$ the model (5.17) has staggered longrange order for $\mu \geqslant \mu_{c}, \beta \geqslant \beta_{\mathrm{c}}$ in the sense that for such $\mu, \beta$ and $\langle\cdots\rangle$ an infinite-volume state

$$
\left\langle P\left(q_{\alpha}=1\right) P\left(q_{\beta}=(-1)^{|\alpha|+|\beta|}\right)\right\rangle>\frac{1}{4}
$$

uniformly in $\alpha, \beta$. 
The first step of the proof is familiar from other models with staggered long-range order, such as the antiferromagnet; namely we flip spins on the odd sublattice. Thus let $v$ be given by (5.15) and define

$$
\begin{aligned}
& U=\prod_{\alpha \in \Lambda_{\text {oda }}} v_{\alpha}{ }^{+} v_{\alpha}{ }^{-} \\
& \tilde{H}=U H U^{-1}
\end{aligned}
$$

Then, since $U q_{\alpha} U^{-1}=(-1)^{|\alpha|} q_{\alpha}$,

$$
\tilde{H}=\tilde{T}+\sum_{\alpha, \beta \in \Lambda} W(\alpha-\beta) q_{\alpha} q_{\beta}-\mu \sum_{\alpha \in \Lambda} q_{\alpha}^{2}
$$

where (for each nearest neighbor pair $\langle\alpha, \beta\rangle, \alpha$ is the even one)

and

$$
\tilde{T}=-\sum_{\langle\alpha, \beta\rangle \in \Lambda}\left(\psi_{\alpha}^{*} \psi_{\beta}^{*}+\psi_{\beta} \psi_{\alpha}\right)
$$

$$
W(\alpha-\beta)=(-1)^{|\alpha-\beta|} V(\alpha-\beta)
$$

We are thus reduced to showing that the $\tilde{H}$ model has long-range order in the unstaggered sense. The kinetic energy term in $\widetilde{H}$ is already in a "ferromagnetic" form. To see that the $W$ term is also, we note:

Proposition 5.7. Suppose that $V$ is a function on $\mathbb{Z}^{v}$ which is RP with respect to reflections between lattice sites. Then $W(\alpha)=(-1)^{|\alpha|} V(\alpha)$ is $\mathrm{RN}$.

Proof. Let $r\left(\alpha_{1}, \ldots, \alpha_{v}\right)=\left(1-\alpha_{1}, \alpha_{2}, \ldots, \alpha_{\nu}\right)$ for $\alpha_{1} \geqslant 1$. Then for any $c_{\alpha}{ }^{\prime}{ }^{\prime}$

$$
\sum_{\substack{\alpha_{1} \geqslant 1 \\ \beta_{1} \geqslant 1}} V(\alpha-r \beta) \bar{c}_{\alpha} c_{\beta} \geqslant 0
$$

Let $d_{\alpha}=(-1)^{|\alpha|} c_{\alpha}$, so that

$$
\sum W(\alpha-r \beta) \bar{d}_{\alpha} d_{\beta}=-\sum V(\alpha-r \beta) \bar{c}_{\alpha} c_{\beta}
$$

since $|\alpha-r \beta| \equiv|\alpha|+|\beta|+1(\bmod 2)$. Thus $(5.20)$ implies that $W$ is RN.

The general framework of Ref. 11 for quantum systems required distinguished algebras $\mathfrak{A}_{+}$and $\mathfrak{A}_{-}$and a morphism $\theta: \mathfrak{A}_{+} \rightarrow \mathfrak{A}_{-}$. The machinery worked smoothly if $\mathfrak{A}_{+}$and $\mathfrak{A}_{-}$commute. For this reason, we make a preliminary Jordan-Wigner (=Klein) transformation to make the left and right commute. Thus we suppose that $\Lambda$ consists of sites $\alpha$ with $\alpha_{1}=-L+1, \ldots$, $L$. For $\alpha_{1} \geqslant 1$, let $\phi_{\alpha}{ }^{ \pm}=\psi_{\alpha}{ }^{ \pm}$and for $\alpha_{1} \leqslant 0$, let $\phi_{\alpha}{ }^{ \pm}=(-1)^{N_{R}} \psi_{\alpha}{ }^{ \pm}$, where

$$
N_{R}=\sum_{\alpha_{1} \geqslant 1} q_{\alpha}
$$


So

$$
(-1)^{N_{R}}=\prod_{\alpha_{1} \geqslant 1} u_{\alpha}{ }^{+} u_{\alpha}^{-}
$$

Notice that $n_{\alpha}{ }^{ \pm}=\left(\phi_{\alpha}{ }^{ \pm}\right)^{*} \phi_{\alpha}{ }^{ \pm}$and that

$$
\tilde{H}=\tilde{T}_{L}+\tilde{T}_{R}+\tilde{T}_{L R}+\sum W(\alpha-\beta) q_{\alpha} q_{\beta}-\mu \sum q_{\alpha}^{2}
$$

where (with $\alpha$ the even member of the pair $\langle\alpha \beta\rangle$ )

$$
\begin{aligned}
\tilde{T}_{L} & =-\sum_{\substack{\langle\alpha, \beta\rangle \\
\alpha_{1}, \beta_{1} \leqslant 0}}\left(\phi_{\alpha}{ }^{*} \phi_{\beta}{ }^{*}+\phi_{\beta} \phi_{\alpha}\right) \\
\tilde{T}_{R} & =-\sum_{\substack{\langle\alpha, \beta\rangle \\
\alpha_{1}, \beta_{1} \geqslant 1}}\left(\phi_{\alpha}{ }^{*} \phi_{\beta}{ }^{*}+\phi_{\beta} \phi_{\alpha}\right) \\
\tilde{T}_{L R} & =-\sum_{\substack{\langle\alpha, \beta\rangle \\
\alpha_{1} \geqslant 1, \beta_{1} \leqslant 0 \text { or } \alpha_{1} \leqslant 0, \beta_{1} \geqslant 1}}\left[\phi_{\alpha}{ }^{*}(-1)^{N_{R}} \phi_{\beta}{ }^{*}+\phi_{\beta}(-1)^{N_{R} \phi_{\alpha}}\right]
\end{aligned}
$$

Let $\mathfrak{A}_{L}$ (resp. $\mathfrak{A}_{R}$ ) denote the real algebra generated by $\left\{\phi_{\alpha}, \phi_{\alpha}{ }^{*}\right\}$ with $\alpha_{1} \leqslant 0$ (resp. $\alpha_{1} \geqslant 1$ ). Motivated by the formula for $\tilde{T}_{L R}$, we define $\theta: \mathfrak{A}_{L} \rightarrow \mathfrak{A}_{R}$ by

$$
\begin{array}{ll}
\theta\left(\phi_{\alpha}\right)=\phi_{r \alpha}(-1)^{N_{R}} & \text { if } \quad(-1)^{|\alpha|}=1 \\
\theta\left(\phi_{\alpha}\right)=(-1)^{N_{R}} \phi_{r \alpha} & \text { if } \quad(-1)^{|\alpha|}=-1
\end{array}
$$

using Proposition 5.4 to extend $\theta$ to a $*$-automorphism (to be able to do this one needs to check that the image under $\theta$ of the $\phi$ 's obeys the CAR). The asymmetry in the definition of $\theta$ comes from the fact that we have taken $\phi_{\beta} \leftrightarrow \phi_{\beta}{ }^{*}$ for $(-1)^{|\beta|}=-1$.

With this definition of $\theta, \theta\left(n_{\alpha}^{ \pm}\right)=n_{r \alpha}^{ \pm}$and by (5.16), for any $A \in \mathfrak{A}_{L}$

$$
\operatorname{Tr}(\theta(A))=\operatorname{Tr} A
$$

By Proposition 5.4(a), $\operatorname{Tr}$ is real on $\mathfrak{A}$, the real algebra generated by $\mathfrak{A}_{R}$ and $\mathfrak{A}_{L}$. Finally by $(5.21),-\tilde{H}$ has the form $A+\theta A+\sum B_{l} \theta\left(B_{l}\right)$, so by the general theory ${ }^{(6,11)}\langle\cdots\rangle$ is RP and moreover

$$
Z\left(h_{\alpha}\right) \leqslant Z\left(h_{\alpha} \equiv 0\right)
$$

where

$$
Z\left(h_{\alpha}\right)=\operatorname{Tr} \exp \left[-\tilde{T}+\sum W(\alpha-\beta)\left(q_{\alpha}-h_{\alpha}-q_{\beta}+h_{\beta}\right)^{2}+\mu^{\prime} \sum q_{\alpha}^{2}\right]
$$

with $\mu^{\prime}$ defined so that when $h_{\alpha} \equiv 0, Z\left(h_{\alpha}\right)$ is the partition function for $\tilde{H}$. In the usual way ${ }^{(6,11)}(5.23)$ leads to an inequality in the Duhamel two-point function (for $k \neq 0$ )

$$
(\hat{q}(k), \hat{q}(-k)) \leqslant-[2 \beta \hat{W}(k)]^{-1}
$$


The proof of Theorem 5.5 is completed as follows: If long-range order is not present, then by (5.24)

$$
\left(q_{0}, q_{0}\right) \leqslant \frac{1}{2}
$$

for $\beta$ large. A contradiction is obtained by using the Falk-Bruch inequality if we show that

$$
\left\langle q_{0}^{2}\right\rangle \rightarrow 1 \quad(\beta, \mu \text { large })
$$

and

$$
\left\langle\left[q_{0},\left[q_{0}, \tilde{H}\right]\right]\right\rangle \rightarrow 0 \quad(\beta, \mu \text { large })
$$

By a direct calculation $\left[q_{0},\left[q_{0}, \tilde{H}\right]\right]$ is a multiple of

$$
\sum_{\substack{\langle\alpha, \beta\rangle \\ \alpha=0 \text { or } \beta=0}} \psi_{\alpha}^{*} \psi_{\beta}^{*}+\psi_{\beta} \psi_{\alpha}
$$

and this has small expectation between all states with $\left\langle q_{0}{ }^{2}\right\rangle$ and $\left\langle q_{\alpha}{ }^{2}\right\rangle$ $(|\alpha|=1)$ close to 1 . Thus (5.25) implies (5.26).

Condition (5.25) can be proven either by thermodynamic considerations or by a chessboard estimate. Here is the thermodynamic proof. As above, write

$$
-\tilde{H}=-\tilde{T}+\sum W(\alpha-\beta)\left(q_{\alpha}-q_{\beta}\right)^{2}+\mu^{\prime} \sum q_{\alpha}^{2}
$$

with $\mu^{\prime}=\mu-$ const. Since $W$ is $\mathrm{RN}$, the maximum value of $-\sum W(\alpha-\beta) \times$ $\left(q_{\alpha}-q_{\beta}\right)^{2}$ occurs with all $q_{\alpha}$ equal, i.e., it is zero. Thus

$$
\langle-\tilde{H} /|\Lambda|\rangle \leqslant \mu^{\prime}\left\langle q_{0}^{2}\right\rangle+2 \nu
$$

On the other hand, using

and

$$
\operatorname{Tr}(1)=4^{|\Lambda|}
$$

$$
\operatorname{Tr}\left\{\exp \left[-\beta H\left(\mu^{\prime}\right)\right]\right\} \geqslant \exp (-\beta\langle a|\tilde{H}| a\rangle)
$$

(where $a$ is the state with all $q_{\alpha}=+1$ ) and convexity of $\ln \operatorname{Tr} e^{-B}$, we see that

$$
\langle-\tilde{H}|| \Lambda \mid\rangle \geqslant \mu^{\prime}-\beta^{-1} \ln 4
$$

Then (5.27) and (5.28) imply that

$$
\left\langle q_{0}^{2}\right\rangle \geqslant 1-\left(\mu^{\prime}\right)^{-1} \beta^{-1} \ln 4-2\left(\mu^{\prime}\right)^{-1} \nu
$$

proving (5.25). This completes the proof of Theorem 5.5 .

\section{SIX-AND EIGHT-VERTEX MODELS}

In this section we will discuss several aspects of planar six- and eightvertex models in zero external field. We remind the reader that for these 
models, the free energy is known exactly ${ }^{(33-35,51,1)}$ (for a comprehensive review of the six-vertex models see Ref. 37). We will leave extensions to 16vertex models and to higher dimensions to the reader's imagination!

The "spins" in this model are assignments of a direction (or arrow) to each bond of a square lattice. Of the 16 possible configurations at each lattice site only the first six (resp. all eight) of those pictured in Fig. 8 are allowed. The basic Hamiltonian is

$$
H=\sum_{i=1}^{6(8)} N_{i} \epsilon_{i}
$$

where $N_{i}$ is the number of vertices of type $i$.

Model 6.1. (Six-vertex F-model; $\epsilon_{1}=\epsilon_{2}=\epsilon_{3}=\epsilon_{4}>\epsilon_{5}=\epsilon_{6}$.) Consider a vertical line midway between lattice sites. We let $\mathfrak{A}_{0}$ denote functions of the arrows that intersect this line and $\mathfrak{A}_{r}\left(\right.$ resp. $\mathfrak{A}_{l}$ ) functions of the arrows on the right (resp. left). We define $\theta: \mathfrak{A}_{+}=\left[\mathfrak{A}_{r} \cup \mathfrak{A}_{0}\right] \rightarrow \mathfrak{A}_{-}=\left[\mathfrak{A}_{r} \cup \mathfrak{A}_{0}\right]$ as follows (here [2] is the algebra generated by $\mathfrak{2})$ : If $\sigma_{i}(i$ a bond $)$ is \pm 1 , depending on whether the arrow points right (resp. up) or left (resp. down) for $i$ horizontal (resp. vertical), then $\theta\left(\sigma_{i}\right)=\sigma_{\theta i}$ for horizontal bonds and $\theta\left(\sigma_{i}\right)=$ $-\sigma_{\theta i}$ for vertical bonds. By the general theory ${ }^{(11)}$ the uncoupled state is RP. Moreover, the induced map from configurations at site $\alpha$ to those at site $\theta(\alpha)$ is $\theta(1)=(3), \theta(2)=(4), \theta(3)=(1), \theta(4)=(2), \theta(5)=(6), \theta(6)=(5)$. Since

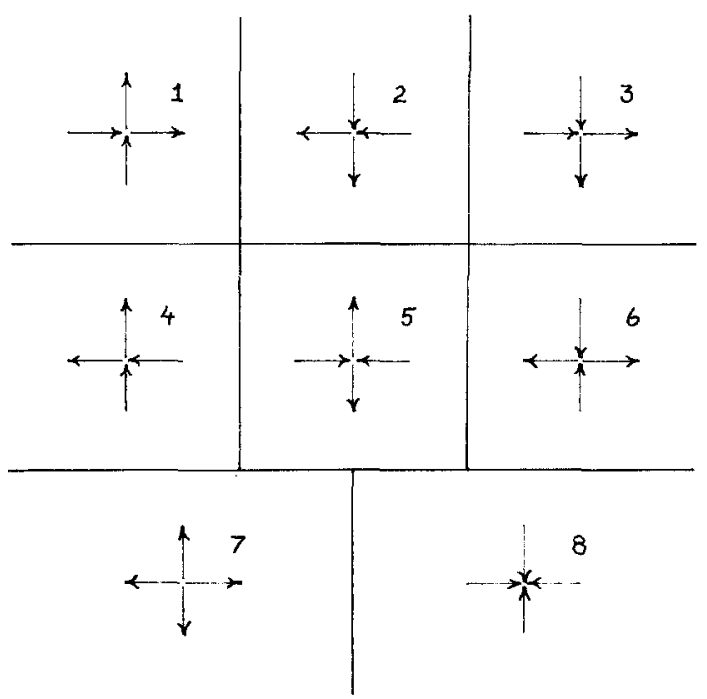

Fig. 8. Configurations of the six- and eight-vertex models. 
the basic interactions only involve spins in $\mathfrak{A}_{+}$or $\mathfrak{A}_{-}, H$ is of the form $A+\theta A$ so long as $\epsilon_{1}=\epsilon_{3}, \epsilon_{2}=\epsilon_{4}, \epsilon_{5}=\epsilon_{6}$. Again by the general theory ${ }^{(11)}$ the interacting state will be RP. If

$$
\epsilon_{1}=\epsilon_{2}=\epsilon_{3}=\epsilon_{4} ; \quad \epsilon_{5}=\epsilon_{6}
$$

we will have RP in both horizontal and vertical planes. In that case, by mimicking the proof of Theorem 4.3 of Ref. 11, we will obtain the following chessboard estimate:

Theorem 6.1. For any function $F$ of the arrows coming into a single site $\mu$, let $\gamma(F)=\left\langle\prod_{\alpha \in \Lambda} \tau_{\alpha}(F)\right\rangle^{1 /|\Lambda|}$, where $\tau_{\alpha}(F)$ is a translate of $F$ if $\alpha_{1}-\mu_{1} \equiv$ $\alpha_{2}-\mu_{2} \equiv 0(\bmod 2)$, a reflected (by $\theta$ as above) translate if $\alpha_{1}-\mu_{1}+1 \equiv$ $\alpha_{2}-\mu_{2} \equiv 0(\bmod 2)$, etc. Then whenever $(6.2)$ holds, we have in a $2 L$ by $2 L$ torus

$$
\left\langle\prod_{\alpha \in \Lambda} F_{\alpha}\right\rangle \leqslant \prod_{\alpha \in \Lambda} \gamma\left(F_{\alpha}\right)
$$

where $F_{\alpha}$ is a function of the arrows at site $\alpha$.

Now suppose that (6.2) holds and $\epsilon_{5}<\epsilon_{1}$. Then there are two ground states, as is easy to see from RP: one obtained from state (5) and reflection, and the other from state (6). They are interchanged by translations of one unit or by flipping all arrows. We can organize contours as follows: let $s_{i}=+1$ if the arrow on bond $i$ is in the direction it would be in one ground state, -1 if it is in the other direction. We now tilt our heads by $45^{\circ}$ and think of the system as an Ising model on a square lattice with spacing $\frac{1}{2} \sqrt{2}$ obtained by putting dots on the midpoints of bonds. Place contours in the standard way for an Ising model, so in terms of the original lattice a contour $\gamma$ is a collection of $|\gamma|$ diagonal segments of length $\frac{1}{2} \sqrt{2}$. The six-vertex condition implies that contours cannot turn at vertices in the original lattice but only at points in the dual lattice of the original lattice (see Fig. 9 for a typical contour). This reduces the number of contours to be counted when one pushes through to an estimate on transition temperatures. Clearly $\gamma$ passes through $\frac{1}{2}|\gamma|$ original lattice sites, and at each site the configuration is forced to be one of

Fig. 9. A typical contour.

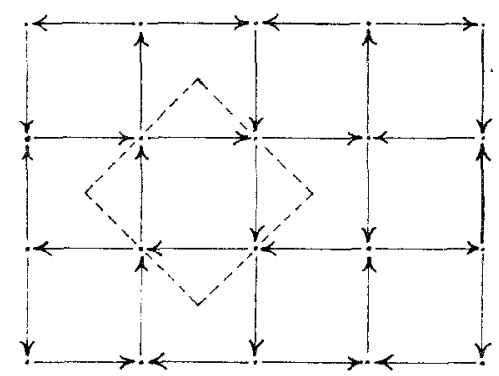


1-4. Moreover, under repeated reflection the projection onto any of the configurations $1-4$ leads to an array like that shown in Fig. 10 with energy per site $\frac{1}{4}\left(\epsilon_{1}+\epsilon_{2}+\epsilon_{3}+\epsilon_{4}\right)$. Thus, by Theorem 6.1,

$$
\operatorname{Prob}(\gamma) \leqslant \exp \left[-\frac{1}{2} \beta|\gamma|\left(\epsilon_{1}-\epsilon_{5}\right)\right]
$$

in the usual way. Thus, since $\left\langle\sigma_{i}\right\rangle=0$ in the periodic state, we obtain:

Theorem 6.2. The Hamiltonian (6.1) with (6.2) and $\epsilon_{1}>\epsilon_{5}$ has at least two equilibrium states for $\beta$ large, with $\left\langle\sigma_{i}\right\rangle \neq 0$ and long-range order.

If $\epsilon_{1} \leqslant \epsilon_{5}$, we obtain a structure very similar to that in Model 2.2 when (2.5) fails. Thus one expects no long-range order; indeed this follows from the known analyticity of the pressure in that case. ${ }^{(37)}$

Model 6.2. (Eight-vertex model.) We have RP in the same planes as in Model 6.1 so long as $\epsilon_{7}=\epsilon_{8}$ in addition to (6.2). If $\epsilon_{5}<\min \left(\epsilon_{1}, \epsilon_{7}\right)$, one obtains phases with the same structure as in the earlier model. Indeed, the only change in the analysis is that contours can now have corners at original lattice sites. If $\epsilon_{7}<\min \left(\epsilon_{1}, \epsilon_{5}\right)$, there are again two ground states and by mimicking the argument in the last model, one obtains multiple equilibrium states. If $\epsilon_{5}=\epsilon_{7}$ (i.e., $\epsilon_{1}=\epsilon_{2}=\epsilon_{3}=\epsilon_{4}=\epsilon$ and $\epsilon_{5}=\epsilon_{6}=\epsilon_{7}=\epsilon_{8}=-\epsilon$ ), then the model is invariant under reversal of all the vertical arrows in any column or all the horizontal arrows in any row, which suggests the absence of any long-range order. This is supported by the following exact calculation of the partition function with free boundary conditions. One can make an arbitrary choice of all vertical arrows and of one horizontal arrow in each row; once this choice has been made, the eight-vertex condition determines all the other horizontal arrows. Moreover, the equality of energies in two sets implies that the energy of a configuration is completely determined by the vertical arrows. Therefore if there are $M$ rows and $L$ columns, we have $Z=2^{M} Q^{L}$, where $Q$ is the partition function for a single column. For each column, the position of the bottom arrow is irrelevant and then we pick up a

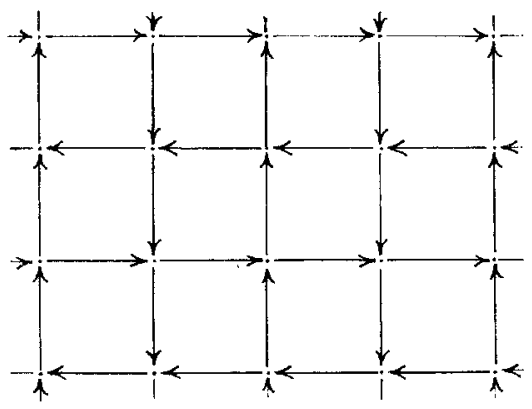

Fig. 10. A universal configuration. 
factor of $\pm \epsilon$, depending on whether each successive arrow is parallel or antiparallel to the preceding one. Thus $Q=2^{M+1}(\cosh \beta \epsilon)^{M}$, so

$$
Z=2^{M+L}(2 \cosh \beta \epsilon)^{L M}
$$

Model 6.3. (Diagonal RP in the generalized $F$ model.) As an exercise in RP, we want to examine when Model 6.1 is RP in a diagonal line through sites. Thus, imagine a diagonal line going from the lower left to the upper right. The geometric $\theta^{\prime}$ on bonds takes vertical bonds into horizontal bonds. We will define $\theta^{\prime}$ on the bond arrows by $\theta^{\prime}(\uparrow)=<, \theta^{\prime}(\downarrow)=\rightarrow, \theta^{\prime}(\rightarrow)=\downarrow$, $\theta^{\prime}(\leftarrow)=\uparrow$, i.e., geometric reflection followed by flip. Then $\theta^{\prime}$ will leave states 3-6 unchanged and will interchange states 1 and 2 . Thus if $\epsilon_{1}=\epsilon_{2}$, the part of the Hamiltonian that does not involve sites on the line will have the form $A+\theta A$. To see whether the remaining piece of $-H$ has the form $\sum B_{i} \theta B_{i}$ we need to look at whether the matrix of statistical weights for a state $\mu$ above and $\nu$ below is positive definite. There are four possibilities for $\mu: a=<^{+}$, $b=\rightarrow^{\uparrow}, c=\rightarrow^{\dagger}, d=\leftarrow^{\uparrow}$. Thus $\theta a={ }_{\uparrow} \rightarrow, \theta b={ }_{\uparrow} \leftarrow, \theta c={ }_{\downarrow} \rightarrow, \theta d={ }_{\uparrow} \leftarrow$, and the matrix $A_{\mu \nu}=\exp [-\beta$ energy of $(\mu \theta \nu)]$ has the form

$$
\left(\begin{array}{llll}
e^{-\beta \epsilon_{5}} & e^{-\beta \epsilon_{2}} & 0 & 0 \\
e^{-\beta \epsilon_{1}} & e^{-\beta \epsilon_{5}} & 0 & 0 \\
0 & 0 & e^{-\beta \epsilon_{3}} & 0 \\
0 & 0 & 0 & e^{-\beta \epsilon_{4}}
\end{array}\right)
$$

So, with the condition $\epsilon_{1}=\epsilon_{2}$ already required, positive-definiteness is equivalent to $\exp \left[-\beta\left(\epsilon_{5}+\epsilon_{6}\right)\right]-\exp \left[-\beta\left(\epsilon_{1}+\epsilon_{2}\right)\right] \geqslant 0$, i.e., $\epsilon_{5}+\epsilon_{6} \leqslant$ $\epsilon_{1}+\epsilon_{2}$. As the general theory (Schoenberg's Theorem) predicts, ${ }^{(11)}$ the condition is independent of $\beta$. Thus:

Theorem 6.3. The six-vertex model is RP with respect to reflections in diagonals as described above if and only if $\epsilon_{1}=\epsilon_{2}$ and $\epsilon_{5}+\epsilon_{6} \leqslant \epsilon_{1}+\epsilon_{2}$ (the conditions $\epsilon_{3}=\epsilon_{4}$ and $\epsilon_{5}=\epsilon_{6}$ are not required).

By using this theorem and reflecting in two diagonal directions, one can extend Theorems 6.1 and 6.2 to the case where $\epsilon_{1}=\epsilon_{2}, \epsilon_{3}=\epsilon_{4}, \epsilon_{5}+\epsilon_{6}<2$ $\min \left(\epsilon_{1}, \epsilon_{3}\right)$.

\section{THE PROJECTED PEIERLS ARGUMENT}

In this section we want to describe a method which allows one to study a $\nu$-dimensional model by considering only contours in a two-dimensional plane. This argument applies to some situations where a naive $\nu$-dimensional contour argument is not applicable. We illustrate the method in a simple case: 
Model 7.1. ( $v$-dimensional Ising model.) Consider the nearest neighbor, spin- $\frac{1}{2}$ Ising model in $\nu(>2)$ dimensions. As usual, let $P_{\alpha}{ }^{ \pm}$be the projection onto $s_{\alpha}= \pm 1$. Suppose we show that $\left\langle P_{\alpha}{ }^{+} P_{\beta}{ }^{-}\right\rangle \leqslant \epsilon$ for all $\alpha, \beta$ in a common two-dimensional plane of the lattice [there are $\left(\begin{array}{c}v \\ 2\end{array}\right)$ types of such planes]. Then for any $\alpha, \gamma$ we can find $\beta_{1}, \ldots, \beta_{k}$ with $k=\left[\frac{1}{2}(\nu-1)\right]$ and the pairs $\left(\alpha, \beta_{1}\right)$, $\left(\beta_{1}, \beta_{2}\right), \ldots,\left(\beta_{k}, \gamma\right)$ in common two-dimensional planes. But since (with $\left.\beta_{0}=\alpha, \beta_{k+1}=\gamma\right)$

$$
\left\langle P_{\alpha}{ }^{+} P_{\gamma}{ }^{-}\right\rangle \leqslant \sum_{j=0}^{k}\left\langle P_{\beta_{j}}^{+} P_{\beta_{j+1}}^{-}\right\rangle
$$

we see that $\left\langle P_{\alpha}{ }^{+} P_{\gamma}{ }^{-}\right\rangle \leqslant(k+1) \epsilon$. Thus a proof of two-dimensional "longrange order" implies full long-range order.

Now let $\alpha, \beta$ lie in a common plane. By considering configurations of the spins in that plane, we can dominate $\left\langle P_{\alpha}{ }^{+} P_{\beta}{ }^{-}\right\rangle$by a sum, over contours $\Gamma$ in that plane, of the probability $P(\Gamma)$ that in that plane all the spins inside $\Gamma$ are plus and those outside $\Gamma$ are minus. But by chessboard estimates, $P(\Gamma) \leqslant$ $\exp (-a|\Gamma|)$ with $a \rightarrow \infty$ as $T \rightarrow 0$. This proves long-range order.

Model 7.2. (The Slawny model.) Take $v=3$ for simplicity and spin $-\frac{1}{2}$ spins. For each $a$, a square face of some cube in the basic lattice $\mathbb{Z}^{3}$, let $t_{a}$ be the product of the spins at the corners of $a$. The Slawny Hamiltonian ${ }^{(50)}$ is

$$
H=\sum_{a \Lambda} t_{a}
$$

The reason for the interest in this Hamiltonian is that is possesses an infinite local symmetry group; namely, flipping all spins in any family of parallel planes leaves $H$ invariant. This implies that there will be infinitely many phases once some kind of long-range order is shown. We remark that, while there are some similarities between the Slawny model and the currently fashionable $\mathbb{Z}_{2}$-gauge model, they are very different. The latter model has symmetries that affect only a finite number of spins, and no long-range order.

Two proofs of such long-range order have been given: in Ref. 50, Slawny used correlation inequalities to compare the model to the two-dimensional Ising model, and in Ref. 28, Holsztyński and Slawny introduced a sophisticated contour argument applicable to this model. Here, we will prove a kind of long-range order. Going from this to the infinite number of states will be left to the reader. Note first that one has RP in planes between lattice sites. Fix a horizontal plane, and for each (original lattice) site $\alpha$ in the plane let $z_{\alpha}$ be the product of the spin at $\alpha$ and the spin directly above $\alpha$. Let $P_{\alpha}{ }^{ \pm}$be the projection onto $z_{\alpha}= \pm 1$. We will show that $\left\langle P_{\alpha}{ }^{+} P_{\gamma}{ }^{-}\right\rangle$is small for $\beta$ sufficiently large, by a two-dimensional contour analysis. In the usual way, the probability of a contour $\Gamma$ will be dominated by $a^{|\Gamma| / 4}$, where $a=\left\langle P_{\text {univ }}\right\rangle_{\Lambda}^{4 / \Lambda \mid}$. 
Here $P_{\text {univ }}$ results from taking $P_{\alpha}^{+} P_{\alpha+\delta}^{-}[\delta=(1,0,0)]$ and making repeated reflections, with a two-element period in the vertical and $\delta$ directions and a one-element period in the third direction. Since in the resulting configuration $1 / 12$ of the squares have $t_{\alpha}=-1$, we obtain $a \rightarrow 0$ as $\beta \rightarrow \infty$. Finally, we note that $\left\langle P_{\alpha}{ }^{+}\right\rangle=\left\langle P_{\alpha}{ }^{-}\right\rangle=\frac{1}{2}$ by the previously mentioned symmetry. This implies long-range order.

Model 7.3. Let us contrive a model where the method of this section works, but where no other method we know will work. Take the Slawny model and make a small (proportional to $\epsilon$ ) perturbation by adding a nearest neighbor antiferromagnetic coupling and a small external field. This perturbation destroys the ferromagnetic properties that make the correlation inequalities work, and the decomposition property of Ref. 28 fails. One can use the Pirogov-Sinai method, but the resulting bound on the transition temperature will go to zero as $\epsilon$ does. By modifying the method of Model 7.2 we obtain a lower bound going to the bound of that model as $\epsilon \downarrow 0$.

\section{THE BALANCED MODEL}

On the lattice $\mathbb{Z}^{v}$ the basic Hamiltonian is

$$
-\beta H=\beta \sum_{\langle\alpha \gamma\rangle} \sigma_{\alpha} \sigma_{\gamma}-\frac{\beta}{2(\nu-1)} \sum_{\langle\alpha \gamma\rangle}^{\prime} \sigma_{\alpha} \sigma_{\gamma}
$$

where the first sum is over all nearest-neighbor pairs and the second sum is over all next-nearest-neighbor pairs. This model entered naturally in our analysis in Ref. 11. If $-1 /[2(\nu-1)]$ is replaced by $\alpha$ in $(8.1)$, then one has $\mathrm{RP}$ about planes between sites if and only if $2|\alpha|(\nu-1) \leqslant 1$, i.e., (8.1) is at the borderline of RP. Moreover, it is at a point of balance between ferromagnetism and antiferromagnetism. The interaction of a single spin with a neighboring hyperplane of all plus spins is zero due to a precise cancellation of the ferromagnetic and antiferromagnetic interactions. For this reason, we call the model the "balanced model."

Model 8.1. (Balanced Ising model; breaking of discrete symmetry.) Here we have nothing definite to report; we will describe what we believe happens. First, there should not be a first-order phase transition in two dimensions: for, if one uses the naive Peierls argument, one finds that only corners of contours are "bad" in that they make positive contributions to the energy. Thus, there are infinitely many contours (namely those with four corners) with the same energy shift, and one expects entropy to overwhelm energy and prevent the occurrence of long-range order.

We believe that in $\nu \geqslant 3$ dimensions, there are at least two phases 
(indeed, as we discuss below, probably exactly two). Various methods have not yet yielded a proof:

(i) The basic chessboard Peierls argument fails for an interesting reason. In finite volume, one must consider three kinds of contours: ones that surround $\alpha$, ones that surround $\gamma$, and ones that wrap around the torus. The first two classes typically have negligible probability as $|\Lambda| \rightarrow \infty$. However, in this case there are contours of zero energy wrapping around the torus, so that the chessboard Peierls argument fails.

(ii) The naive Peierls argument comes very close to working: use plus boundary conditions and put in conventional contours $\Gamma$ (which are twodimensional if $\nu=3$ ). The set of "edges" of $\Gamma$ is what counts. Given a set $e$ of edges, of length $|e|$, the number of $\Gamma$ with those edges is easily bounded by $A^{|e|}$. Moreover, the set of connected edge graphs that surround a vertex $\alpha$ is easily bounded by $B C^{|e|}$. The problem is that the set of edges need not be connected. Indeed, if $\Gamma$ is a large cube of side $l$ with $l$ unit cubes removed from the surface, $|e| \leqslant 12 l+12 l$, but the number of ways of removing the cubes goes like

$$
\left(\begin{array}{c}
l^{2} \\
l
\end{array}\right) \simeq e^{a l \ln l}
$$

which overwhelms the energy factor.

(iii) The analysis in (ii) suggests that we try a procedure of removing a single connected piece of edge contour. This is essentially the method that Holsztyński and Slawny ${ }^{(28)}$ use. The problem is that in their language the decomposition property fails, and it is far from clear how to make this procedure work.

(iv) One can try the projected Peierls argument of Section 7. As in the analysis of the Slawny model (Model 7.2), one easily shows that with $P^{ \pm}$as in that model

$$
\left\langle P_{\alpha}{ }^{+} P_{\nu}{ }^{-}\right\rangle \rightarrow 0 \quad \text { as } \beta \rightarrow \infty
$$

The difference from the Slawny model is the following: in that model, $\left\langle P_{\alpha}{ }^{+}\right\rangle=\left\langle P_{\alpha}{ }^{-}\right\rangle=\frac{1}{2}$ by symmetry, so (8.2) implies that long-range order occurs. In the case at hand, it seems quite likely that $\left\langle P_{\alpha}{ }^{-}\right\rangle \rightarrow 0$ as $\beta \rightarrow \infty$, preventing long-range order of the $t_{\alpha}=\sigma_{\alpha} \sigma_{\alpha+\delta}$. Of course, $\left\langle P_{\alpha}^{-}\right\rangle$small means that neighboring spins tend to have the same sign, which strongly suggests that there is long-range order of the $\sigma_{\alpha}$ 's.

(v) The following chessboard Peierls argument works, modulo a geometrical lemma which we have not been able to prove. Consider four sites $\alpha_{1}, \ldots, \alpha_{4}$ forming the corners of a rectangle $R$ with sides parallel to the $x, y$ axes. We wish to show that $\left\langle\prod \sigma_{\alpha_{i}}\right\rangle>\frac{1}{2}$ for large $\beta$, uniformly in $R$. This will imply long-range order of some sort, although not necessarily a spontaneous magnetization. The geometrical result is the following: 
Conjecture. In any configuration with $\sigma_{\alpha_{1}} \sigma_{\alpha_{2}} \sigma_{\alpha_{3}} \sigma_{\alpha_{4}}=-1$ there is some $L$ and some connected set of contour edges of length $L$ within a distance $2 L$ of one of the $\alpha_{i}$.

Given this conjecture, the inequality follows by chessboard estimates and easy bounds on the number of connected sets of edges of length $L$ in a given volume. The conjecture would follow in turn from a second conjecture:

Conjecture. In any configuration as above, there is a connected component $E$ of contour edges such that either $E$ is unbounded and intersects $R$, or $E$ is bounded and the smallest box with sides parallel to the axes containing $E$ also contains one of the $\alpha_{i}$.

While these conjectures seem reasonable, we have not been able to prove them.

(vi) One can try infrared bounds. As we shall see, these only work if $\nu \geqslant 5$, where indeed one can prove that some long-range order occurs.

The Hamiltonian (8.1) has an infinity of ground states; indeed, any array of hyperplanes with constant $\sigma_{\alpha}$ in each hyperplane is a ground state. On this basis, one might expect an infinity of phases, but we believe this is unlikely, for the ground states are not related by a symmetry. For this reason, one should expect the need for small external fields to manifest the instability associated with the ground state. Indeed, adding a term $\beta C \sum_{\alpha} t_{\alpha}$ to the $H$ of (8.1), one can still prove (8.2) uniformly in $C$ small. On the other hand, it is easy to see that for suitable $C_{0}$ and $\beta_{0}$

$$
\left\langle P_{\alpha}{ }^{ \pm}\right\rangle<\frac{1}{2}
$$

for $\mp C \geqslant C_{0} \beta^{-1}, \beta>\beta_{0}$. This means that there will be multiple phases at a point $C(\beta)$ with $|C(\beta)| \leqslant C_{0} \beta^{-1}$. More generally one expects, given $n$, to be able to add a suitable small external field and obtain at least $n$ phases.

Model 8.2. (Balanced classical Heisenberg model; breaking of continuous symmetry.) The spin wave energy in (8.1) is

$$
E_{p}=\sum_{i=1}^{v} \cos p_{i}-\frac{1}{2(\nu-1)} \sum_{i=1}^{v} \sum_{j>i}\left[\cos \left(p_{i}+p_{j}\right)+\cos \left(p_{i}-p_{j}\right)\right]
$$

There are several important features of (8.3):

(a) $E_{p} \geqslant 0 ; E_{p}=0$ if and only if at least $\nu-1$ of the $p_{i}$ vanish.

(b) $E_{p} \sim p^{4}$ for $p$ near $0 ; E_{p} \sim\left(p-p_{0}\right)^{2}$ for $p_{0} \neq 0$, but $\nu-1 \mathrm{com}-$ ponents of $p_{0}$ zero.

For $v \geqslant 5, \int E_{p}^{-1} d^{v} p<\infty$, so that the FSS method ${ }^{(17)}$ of infrared bounds implies that the two-point function is a measure with some concentration on the "manifold" of zeros of $E_{p}$. This in turn implies the existence of multiple phases. This argument also applies to the classical Heisenberg model 
[ $\sigma_{\alpha} \sigma_{\gamma}$ in (8.1) replaced by $\sigma_{\alpha} \cdot \sigma_{\gamma}, \sigma$ a unit vector]. If our picture (in the discrete case) of instability but no infinite number of qualitatively different phases is correct here too, then one expects the singularity in the two-point function to occur only at $p=0$.

\section{APPENDIX. A TOPOLOGICAL THEOREM}

In our discussion of multiphase results we required a theorem (Theorem A3 below) which is a special case of Theorem 13 of Brezis. ${ }^{(2)}$ Since this reference is of limited availability, we provide here for the reader's convenience a proof patterned after ideas from ${ }^{(2)}$ and also some ideas contained in a proof given us by J. Mather at a time when we were unaware of Brezis' paper.

Lemma A1. (Variational inequality of Hartman and Stampacchia.) ${ }^{(24)}$ Let $A$ be a continuous map from $C$, a compact convex subset of $\mathbb{R}^{n}$, to $\mathbb{R}^{n}$. Then there exists $u \in C$ so that $(A(u), u-v) \leqslant 0$ for all $v \in C$.

Proof (Brezis, ${ }^{(2)}$ Lemma 4). Since $C$ is compact and convex, for any $z \in \mathbb{R}^{n}$ there exists a unique point $P(z) \in C$ with

$$
\|P(z)-z\|=\inf _{x \in C}\|x-z\|
$$

Notice that $P(z)=u$ if and only if for all $v \in C,\left.(d / d \lambda)\|u+\lambda(v-u)-z\|\right|_{\lambda=0}$ $\geqslant 0$, which holds if and only if $(u-z, v-u) \leqslant 0$ for all $v \in C$. Now let $T: C \rightarrow C$ be defined by

$$
T(u)=P(u-A(u))
$$

By the Brouwer fixed-point theorem, ${ }^{(53)}$ there is some $u$ with $T(u)=u$, i.e., with $(u-(u-A(u)), v-u) \leqslant 0$ for all $v \in C$.

Theorem A2. Let $\Delta_{n}$ be the canonical $n$-simplex $\left\{\left(t_{0}, \ldots, t_{n}\right) \in \mathbb{R}^{n+1}\right.$ : $\left.t_{i} \geqslant 0, \sum t_{i}=1\right\}$ and let $\left\{E_{j}\right\}_{j=1}^{J_{n}}\left(J_{n}=2^{n+1}-2\right)$ be the family of generalized edges (subsets where some nonempty subset $\left\{t_{i}\right\}_{i \in I_{j}}$ of the $t$ 's are all zero and the other $t$ 's are all nonzero). Let $F: \Delta_{n} \rightarrow \Delta_{n}$ be a continuous map with $\operatorname{dist}\left(F(u), E_{j}\right)<(n+1)^{-1}$ for all $u \in E_{j}$ and all $j$. Then there exists some $u \in \Delta_{n}$ with

$$
F(u)=x_{0} \equiv\left(\frac{1}{n+1}, \ldots, \frac{1}{n+1}\right)
$$

Proof. Let $A(u)=F(u)-x_{0}$. By Lemma $A 1$, there exists $u_{0} \in \Delta_{n}$ with $\left(F\left(u_{0}\right)-x_{0}, u_{0}-v\right) \leqslant 0$ for all $v \in \Delta_{n}$. If $u_{0}$ is an interior point of $\Delta_{n}$, then $F\left(u_{0}\right)-x_{0}$ must be zero, so the theorem is proven. Since $\partial \Delta_{n}=\cup_{j} E_{j}$, we will be done if we can use the condition $\operatorname{dist}\left(F(u), E_{j}\right)<(n+1)^{-1}$ to prove that $u_{0}$ cannot lie in any $E_{j}$. Consider first an $E_{j}$ with a single zero coordinate. 
Fig. 11

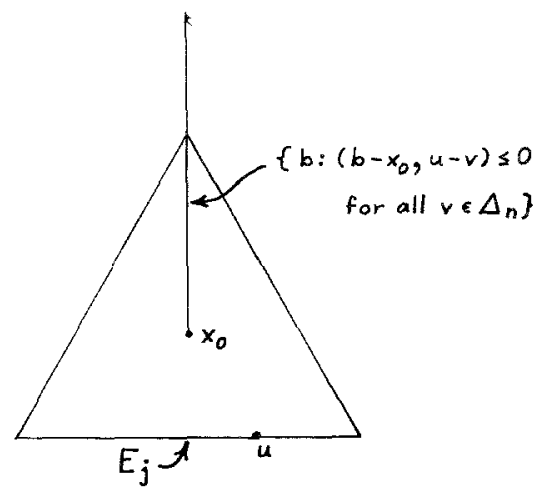

Fix $u \in E_{j}$. Then $\left\{a: \sum a_{i}=0,(a, u-v) \leqslant 0\right.$ for all $\left.v \in \Delta_{n}\right\}$ is a half-line perpendicular to the face $E_{j}$. Thus $\left(F(u)-x_{0}, u-v\right) \leqslant 0$ implies that $F(u)$ must lie in a half-line moving up from $x_{0}$ (see Fig. 11), and this is not allowed by the condition $\operatorname{dist}\left(F(u), E_{j}\right)<(n+1)^{-1}$. A similar geometric argument works for each face.

Theorem A3. (A rather special case of Theorem 13 of Ref. 2). Let $F$ be a map of $\Delta_{n}$ into convex subsets of $\Delta_{n}$ so that $\left\{(x, u): x \in \Delta_{n}, u \in F(x)\right\}$ is closed. Suppose that for some $A<(n+1)^{-1}$ and each generalized edge $E_{j}$ we have that $x \in E_{j}$ and $u \in F(x)$ implies that $\operatorname{dist}\left(u, E_{j}\right) \leqslant A$. Then there exists $u \in \Delta_{n}$ so that

$$
x_{0}=\left(\frac{1}{n+1}, \ldots, \frac{1}{n+1}\right) \in F(u)
$$

Proof. Suppose for each $\delta$ we find $F_{\delta}: \Delta_{n} \rightarrow \Delta_{n}$, so that for $\operatorname{dist}\left(u, \partial \Delta_{n}\right)>\delta$, $\operatorname{dist}\left(F_{\delta}(u), F(u)\right)<\delta$. Then by Theorem A2 we can find, for $\delta$ small, $u_{\delta}$ such that $\operatorname{dist}\left(u_{\delta}, \partial \Delta_{n}\right)>\delta$ and $\operatorname{dist}\left(x_{0}, F\left(u_{\delta}\right)\right)<\delta$. Thus by the compactness of $\Delta_{n}$ there is $u$ such that $x_{0} \in F(u)$. Let $G(u)$ be the barycenter of $F(u)$ for $u \in \Delta_{n}$, and otherwise $G(u)=P(u)$, the nearest point to $u$ in $\Delta_{n} . G$ is not continuous, but if $j_{\epsilon}$ is a smooth function supported in $\{x$ : $\operatorname{dist}(x, 0)<\epsilon\}$ and $\int j_{\epsilon} d^{n+1} x=1$, then $G_{\epsilon}=G * j_{\epsilon}$ is smooth and $\operatorname{dist}\left(G_{\epsilon}(u), F(u)\right)$ is small by the hypotheses on $F$. Choosing $F_{\delta}=G_{\epsilon(\delta)}$ for suitable $\epsilon(\delta)$, the result follows.

Remark. Here is an alternate proof of Theorem A2 which depends on the following lemma of Sperner ${ }^{(58)}$ :

Lemma A4. Let $\Delta_{n}$ be an $n$-simplex with $(n-1)$-dimensional faces $G_{1}, \ldots, G_{n+1}$. Let $B_{1}, \ldots, B_{n+1}$ be closed subsets of $\Delta_{n}$ with $B_{i} \cap G_{i}=\varnothing$. Then either $\cap_{j} B_{j} \neq \varnothing$ or $\cap_{j}\left(\Delta_{n} \mid B_{j}\right) \neq \varnothing$. In particular, if $\cup_{j} B_{j}=\Delta_{n}$, then $\cap_{j} B_{j} \neq \varnothing$. 
Alternate proof of Theorem A2. Let $F_{i}(u)$ denote the coordinates of $F(u)$. Let

$$
B_{j}=\left\{u: \quad F_{j}(u) \geqslant 1 /(n+1)\right\}
$$

Then $\cup_{j} B_{j}=\Delta_{n}$. By hypothesis, $B_{j} \cap G_{j}=\varnothing$, so by the lemma $\cap_{j} B_{j} \neq \varnothing$.

\section{ACKNOWLEDGMENTS}

It is a pleasure to thank $\mathrm{H}$. Brézis, J. Bricmont, D. Brydges, O. J. Heilmann, J. Mather, J. Slawny, and T. Spencer for valuable discussions. One of us (R. I.) would like to thank A. Jaffe for the hospitality of the Harvard Physics Department, where some of this work was done.

\section{REFERENCES}

1. R. J. Baxter, Phys. Rev. Lett. 26:832 (1971).

2. H. Brezis, Séminaire Choquet 6 (18) (1966/67).

3. J. Bricmont, J. L. Lebowitz, and C. E. Pfister, in preparation.

4. D. Brydges, Comm. Math. Phys. 58:313 (1978).

5. R. L. Dobrushin, Func. Anal. Appl. 2: 302 (1968).

6. F. J. Dyson, E. H. Lieb, and B. Simon, J. Stat. Phys. 18:335 (1978).

7. M. E. Fisher, unpublished.

8. J. Fröhlich, Acta Phys. Aust. Suppl. XV:133 (1976).

9. J. Fröhlich, Bull. Am. Math. Soc. 84:165 (1978).

10. J. Fröhlich, Princeton University Lectures (Spring 1977).

11. J. Fröhlich, R. B. Israel, E. H. Lieb, and B. Simon, Comm. Math. Phys. 62:1 (1978).

12. J. Fröhlich, R. B. Israel, E. H. Lieb, and B. Simon, in preparation.

13. J. Fröhlich and E. H. Lieb, Comm. Math. Phys. 60:233 (1978).

14. J. Fröhlich and Y. M. Park, Comm. Math. Phys. 59:235 (1978).

15. J. Fröhlich and Y. M. Park, in preparation.

16. J. Fröhlich and B. Simon, Ann. Math. 105:493 (1977).

17. J. Fröhlich, B. Simon, and T. Spencer, Comm. Math. Phys. 50:79 (1976).

18. J. Fröhlich and T. Spencer, Cargèse Lectures (1976).

19. J. Fröhlich and T. Spencer, in preparation.

20. G. Gallavotti and J. L. Lebowitz, J. Math. Phys. 12:1129 (1971).

21. G. Gallavotti and S. Miracle-Solé, Phys. Rev. B 5:2555 (1972).

22. J. Glimm, A. Jaffe, and T. Spencer, Comm. Math. Phys. 45:203 (1975).

23. F. Guerra, L. Rosen, and B. Simon, Ann. Math. 101:111 (1975).

24. P. Hartman and G. Stampacchia, Acta Math. 115:271 (1966).

25. O. J. Heilmann, Comm. Math. Phys. 36:91 (1974); Lett. Nuovo Cimento 3:95 (1972).

26. O. J. Heilmann and E. H. Lieb, J. Stat. Phys., to appear.

27. O. J. Heilmann and E. Praestgaard, J. Stat. Phys. 9:23 (1973); J. Phys. A 7:1913 (1974).

28. W. Holsztyński and J. Slawny, unpublished (1976), and in preparation.

29. F. Hund, Z. Physik 94:11 (1935).

30. R. B. Israel, in Statistical Physics-'Statphys 13,' D. Cabib, C. G. Kuper, and I. Riess, eds., Ann. Israel Phys. Soc. 2:528 (1978). 
31. Q. C. Johnson and D. H. Templeton, J. Chem. Phys. 34:2004 (1961).

32. D. Kim and C. J. Thompson, J. Phys. A 9:2097 (1976).

33. E. H. Lieb, Phys. Rev. 18:692 (1967).

34. E. H. Lieb, Phys. Rev. 18:108 (1967).

35. E. H. Lieb, Phys. Rev. 19:103 (1967).

36. E. H. Lieb, in Springer Lecture Notes in Physics, No. 80 (1978), pp. 59-67.

37. E. H. Lieb and F. Y. Wu, in Phase Transitions and Critical Phenomena, C. Domb and M. S. Green, eds. (Academic Press, 1972), Vol. 1, p. 331.

38. E. Madelung, Phys. Z. 19:524 (1978).

39. V. A. Malyshev, Comm. Math. Phys. 40:75 (1975).

40. N. D. Mermin, J. Math. Phys. 8:1061 (1967).

41. R. Peierls, Proc. Camb. Phil. Soc. 32:477 (1936).

42. S. A. Pirogov and Ja. G. Sinai, Func. Anal. Appl. 8:21 (1974).

43. S. A. Pirogov and Ja. G. Sinai, Theor. Math. Phys. 25:1185 (1975).

44. S. A. Pirogov and Ja. G. Sinai, Theor. Math. Phys. 26:39 (1976).

45. Y. Sakamoto and U. Takahasi, J. Chem. Phys. 30:337 (1959).

46. M. Schick and R. B. Griffiths, J. Phys. A 10:2123 (1977).

47. E. Seiler and B. Simon, Ann. Phys. 97:470 (1976).

48. B. Simon, in Mathematics of Contemporary Physics, R. Streater, ed. (Academic Press, 1972), pp. 18--76.

49. B. Simon, in Statistical Physics-'Statphys 13,' D. Cabib, C. G. Kuper, and I. Riess, eds., Ann. Israel Phys. Soc. 2:287 (1978).

50. J. Slawny, Comm. Math. Phys. 35:297 (1974).

51. B. Sutherland, Phys. Rev. Lett. 19:103 (1967).

52. M. P. Tosi, Solid State Phys. 16:1 (1970).

53. J. W. Vick, Homology Theory; An Introduction to Algebraic Topology (Academic Press, 1973).

54. P. P. Ewald, Ann. Phys. 64:253 (1921).

55. H. M. Evjen, Phys. Rev. 39:675 (1932).

56. F. C. Frank, Phil. Mag. 41:1287 (1950).

57. M. H. Cohen and F. Keffer, Phys. Rev. 99:1128 (1955).

58. E. Sperner, Abh. Math. Sem. Univ. Hamburg 6:265 (1928). 